\title{
Performance of horizontal wells in composite tight gas reservoirs considering stress sensitivity
}

\author{
Kui Zhao $^{\circledR *}$, Peng Du \\ Qingdao Jari Industry Control Technology, Qingdao 266000, P. R. China
}

(Received June 4, 2019; revised July 25, 2019; accepted July 27, 2019; available online August 9, 2019)

\section{Citation:}

Zhao, K., Du, P. Performance of

horizontal wells in composite tight gas reservoirs considering stress sensitivity.

Advances in Geo-Energy Research, 2019, 3(3): 287-303, doi:

10.26804/ager.2019.03.07.

Corresponding author:

*E-mail: zhaokui716@163.com

Keywords:

Heterogeneity

stress-sensitivity

unsteady fluid exchange

mathematical model

flow regimes

sensitivity analysis

\begin{abstract}
:
Tight gas reservoir (TGR) plays an important role in unconventional oil and gas resources. The existing seepage models for TGR rarely consider the effects of heterogeneity, stresssensitivity, and the unsteady fluid exchange between matrix and fracture. Heterogeneity is common for tight gas reservoir which should be carefully considered in geological model. The stress-sensitivity effect of fracture is an important factor influencing the transient flow behavior of TGR. Ultra-low porosity and permeability cause the unsteady flow between matrix and fractures systems. So this paper introduced a mathematical model for the horizontal well in a dual-porosity composite tight gas reservoir with considering the stress-sensitivity effect and unsteady flow between matrix and fractures systems. Some mathematical methods including the finite Fourier cosine transform, perturbation technique, Laplace transform, superposition principle, Stehfest numerical inversion algorithm are used to solve the nonlinear partial differential equation. Different flow regimes are divided based on pressure transient analysis curves. The sensitivity analysis of related parameters is studied according to pressure transient analysis and rate transient analysis curves. The presented model and obtained results in this paper give better understanding on pressure and rate transient behaviors of composite TGR.
\end{abstract}

\section{Introduction}

With the decline of production from conventional sources, economically producing gas from unconventional sources, such as tight gas, attracts more attention today. Tight gas is an unconventional natural gas resource that is valued by various countries in the world (Law et al., 2002; Holditch et al., 2006; Naik, 2008). The United States has been developing tight gas exploration technology since the late 1970s and has discovered more than 20 tight gas fields. In China, abundant tight gas is distributed in Sichuan basin and Ordos basin, and it is estimated that the tight gas production of China will reach 80 billion cubic meters by 2020 (Jia et al., 2012; Li et al., 2017). For China and the rest of the world, tight gas reservoirs have become an important alternative energy source for conventional oil and gas resources.

Tight gas exists in underground reservoirs with microdarcyrange permeability, with the existence of a large number of natural fractures. Horizontal well is widely used in the development of tight gas as an effective stimulation. Many scholars have carried out related researches on naturally fractured reservoir based on continuous assumption some dual media models were generated, including Warren-Root (1963) model, Kazemi (1968) model and de Swaan (1976) model. Later, some scholars began to introduce fractal models to study the characteristics of fractured reservoirs, where properties are scaled with distance to some reference point in the domain (Chang et al., 1990; Albinali et al., 2016; de Swaan, 2016). In recent years, numerical simulation attracts researchers' attention. It is able to consider more complicate situation of unconventional reservoirs using meshed numerical models (Wu et al., 2014; Noetinger, 2015; Ngo et al., 2017; Zhang et al., 2017; Ding et al., 2018). However, analytical and semianalytical methods still need to be valued for their fast and low-cost advantages.

For production performance of horizontal wells in naturally fractured reservoirs, Göktas et al. (2000) presented a comparative study of openhole and cased horizontal well completions in thin bedded, tight sand gas reservoirs. Freeman et al. (2009) developed a numerical model of microscale flow behavior in tight gas and shale gas systems, which was capable of characterizing flow behavior in micro- and nano-scale porous media. Brown et al. (2011) presented an analytical trilinearflow model to simulate the performance of fractured horizontal 
wells in unconventional shale reservoirs. Nie et al. (2012) investigated the dual permeability flow issue for horizontal wellproduction in a naturally fractured dual-porosity reservoir, they considered the fracture and matrix systems as the flow pathway connected with wellbore. Xu et al. (2013) developed analytical solutions for shale gas/tight gas reservoirs with multi-stage fractured horizontal well accounting for the unstimulated region as a dual porosity system. Kuchuk et al. (2014) investigated rate-transient behavior of dual-porosity and discrete naturally fractured reservoirs using semi-analytical solutions. Li et al. (2017) deduced a dual-porosity model for a horizontal well in tight gas reservoirs, the fluid exchange between matrix systems and fracture systems is considered to be a pseudo-steady state process.

Actual tight gas reservoir (TGR) are heterogeneous and a lot of factors can cause this issue, such as the geologic discontinuities and some production technologies. The model of this article is capable of understanding the behavior of composite TGR. The pressure transient analysis (PTA) and rate transient analysis (RTA) are always used in which the relationship of dimensionless pressure and rate with dimensionless time can be evaluated. For horizontal wells in composite reservoirs, Wang et al. (1997) derived an effective well diameter model of horizontal well in radial composite reservoirs by Laplace transform and superposition principle. Shi et al. (2012) studied a transient well testing model of horizontal well in composite reservoirs. They considered the porous media as homogeneous single porosity media. The method of separation of variables and Laplace transform were utilized. Ozkan et al. (1987) took advantage of Green's function and source-sink theory to research the transient pressure behavior of horizontal well in composite reservoirs. Ezulike et al. (2012) constructed a 3D seepage model for linear composite reservoirs, semianalytical solution for the drawdown response in composite clastic systems separated by a leaky fault was developed. Jiang et al. (2015) recognized the defects of the existing horizontal well models of composite reservoirs, the situation when the horizontal well passed through the inner region in their model is taken into account. For unconventional reservoirs, $\mathrm{Xu}$ et al. (2015) studied the production performance analysis for composite shale gas reservoir. Wei et al. (2017) introduced the mathematical model of production performance analysis for horizontal wells in composite coal bed methane reservoir. As for stress-sensitivity effect of TGR, so many scholars have proved the existence of the stress-sensitivity effect of tight oil and gas reservoirs based on experimental research, and the existence of this effect must affect the production dynamics of tight gas (Shi et al., 2001; Zhang et al., 2004; Yu et al., 2007; Zhu et al., 2013). However, only few scholars take this scenario into account in their mathematical models.

This paper summarizes the advantages and disadvantages of previous scholars, taking into account the following practical factors: (1) the tight gas reservoir is heterogeneous, which is considered as a radial composite model in this article; (2) considering the ultra-low permeability of TGR, the fluid exchange of matrix and fracture systems should not be considered as pseudo-steady state process, and the unsteady state flow model in matrix is established in this paper; (3) stress-sensitivity effect of TGR is coupled into this mathematical model. The pressure transient analysis and rate transient analysis curves are analyzed in detail. The goal is to demonstrate the PTA and RTA results for the better understanding of the production performances in tight gas reservoirs' development.

\section{Physical model}

The schematic diagram for horizontal well in composite TGR is shown in Fig. 1. The composite gas reservoir is divided into inner and outer region, both inner region and outer region are seen as dual porosity media and are formed by matrix and natural fracture systems. The model assumes: (1) the reservoir is horizontal with uniform thickness $h$ and original pressure $p_{i}$; (2) the inner region radius is $r_{1}$ and the outer region is infinite, the top and bottom of the reservoir are closed; (3) the formation parameters of two regions are distinguished, for the fracture system of inner region, the horizontal initial permeability is $k_{f h i 1}$, the vertical initial permeability is $k_{f v i 1}$, the compressibility is $C_{t f 1}$, the porosity is $\phi_{f 1}$, for the matrix system, the permeability is $k_{m 1}$, the compressibility is $C_{t m 1}$, the porosity is $\phi_{m 1}$; thus for the outer region, the parameters are $k_{f h i 2}, k_{f v i 2}, C_{t f 2}, \phi_{f 2}, k_{m 2}, C_{t m 2}, \phi_{m 2} ;$ (4) the influence of
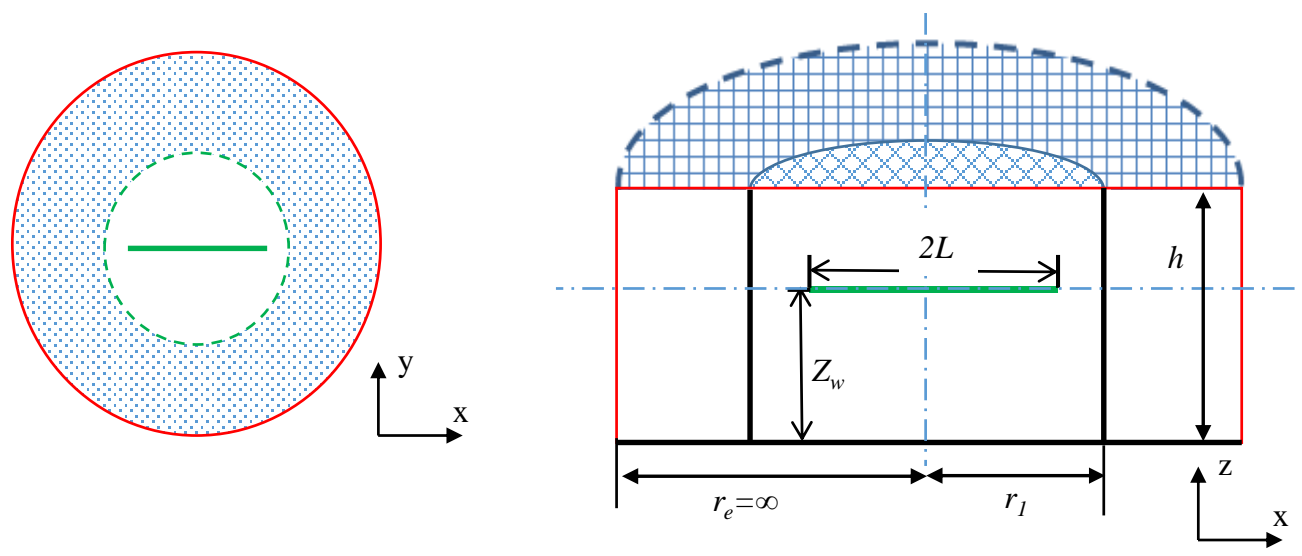

Fig. 1. Horizontal well in composite TGR. 

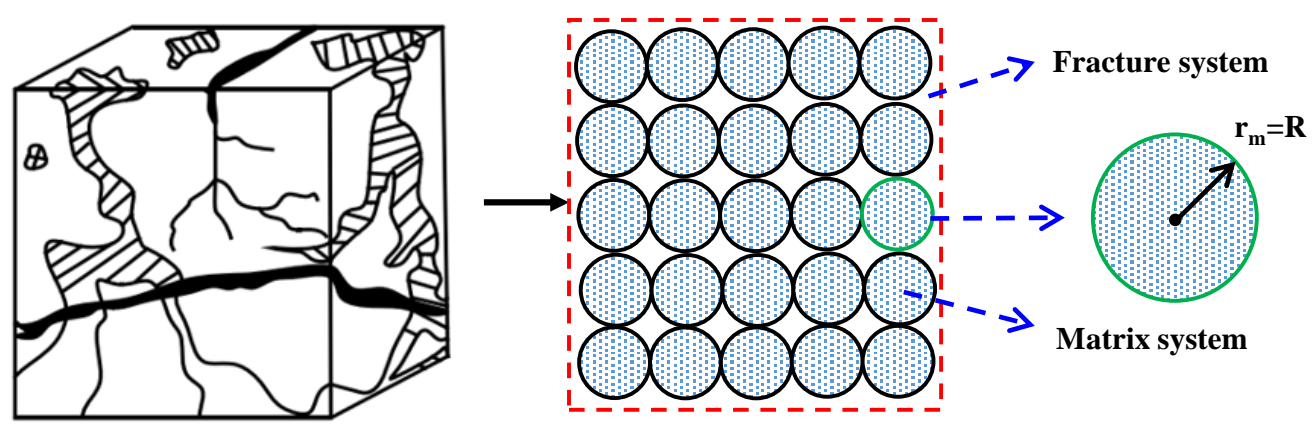

Fig. 2. Schematic of matrix and fracture systems, the first part is revised from Warren and Root (1963).

gravity and capillary forces on fluid flow in both regions is ignored; (5) the fluid properties in both regions are different, the underground flow obey isothermal Darcy seepage; (6) a horizontal well located in inner region totally produces at a constant rate of $q_{s c}$; (7) the matrix blocks are spherical, the schematic diagram of matrix and fracture systems is shown in Fig. 2, matrix blocks are disconnected to wellbore.

\section{Mathematical model}

For the matrix system of inner region, with the consideration of unsteady flow, the partial differential equation (PDE) in the matrix blocks can be obtained as follows from the continuity equation, motion equation and state equations:

$$
\frac{1}{r_{m 1}^{2}} \frac{\partial}{\partial r_{m 1}}\left(r_{m 1}^{2} k_{m 1} \frac{p_{m 1}}{\mu_{1} Z_{1}} \frac{\partial p_{m 1}}{\partial r_{m 1}}\right)=\phi_{m 1} C_{t m 1} \frac{p_{m 1}}{Z_{1}} \frac{\partial p_{m 1}}{\partial t}
$$

Initial condition:

$$
p_{m 1}\left(r_{m 1}, 0\right)=p_{i}
$$

Inner boundary condition:

$$
\left.\frac{\partial p_{m 1}}{\partial r_{m 1}}\right|_{r_{m 1}=0}=0
$$

Outer boundary condition:

$$
\left.p_{m 1}\right|_{r_{m 1}=R_{1}}=p_{f 1}
$$

For the fracture system of inner region, with the consideration of stress sensitivity, the PDE can be expressed as follows:

$e^{\gamma\left(p_{f 1}-p_{i}\right)}\left[\frac{1}{r} \frac{\partial}{\partial r}\left(k_{f h i 1} r \frac{p_{f 1}}{\mu_{1} Z_{1}} \frac{\partial p_{f 1}}{\partial r}\right)+\frac{\partial}{\partial z}\left(k_{f v i 1} \frac{p_{f 1}}{\mu_{1} Z_{1}} \frac{\partial p_{f 1}}{\partial z}\right)\right]$ $+q_{1}^{*}=\phi_{f 1} C_{t f 1} \frac{p_{f 1}}{Z_{1}} \frac{\partial p_{f 1}}{\partial t}$

Analogously, the mathematical model for matrix system of outer region can be expressed as follows:

$$
\frac{1}{r_{m 2}^{2}} \frac{\partial}{\partial r_{m 2}}\left(r_{m 2}^{2} k_{m 2} \frac{p_{m 2}}{\mu_{2} Z_{2}} \frac{\partial p_{m 2}}{\partial r_{m 2}}\right)=\phi_{m 2} C_{t m 2} \frac{p_{m 2}}{Z_{2}} \frac{\partial p_{m 2}}{\partial t}
$$

Initial condition:

$$
p_{m 2}\left(r_{m 2}, 0\right)=p_{i}
$$

Inner boundary condition:

$$
\left.\frac{\partial p_{m 2}}{\partial r_{m 2}}\right|_{r_{m 2}=0}=0
$$

Outer boundary condition:

$$
\left.p_{m 2}\right|_{r_{m 2}=R_{2}}=p_{f 2}
$$

For the fracture system of outer region, with the consideration of stress sensitivity, the PDE can be expressed as follows:

$$
\begin{aligned}
& e^{\gamma\left(p_{f 2}-p_{i}\right)}\left[\frac{1}{r} \frac{\partial}{\partial r}\left(k_{f h i 2} r \frac{p_{f 2}}{\mu_{2} Z_{2}} \frac{\partial p_{f 2}}{\partial r}\right)+\frac{\partial}{\partial z}\left(k_{f v i 2} \frac{p_{f 2}}{\mu_{2} Z_{2}} \frac{\partial p_{f 2}}{\partial z}\right)\right] \\
& +q_{2}^{*}=\phi_{f 2} C_{t f 2} \frac{p_{f 2}}{Z_{2}} \frac{\partial p_{f 2}}{\partial t}
\end{aligned}
$$

The variables $q_{j}^{*}(j=1,2)$ is the unsteady-state interporosity flow rate of the gas from the matrix to the fractures, which is:

$$
q_{j}^{*}=-\left.\frac{3}{R_{j}} k_{m j} \frac{p_{m j}}{\mu_{j} Z_{j}} \frac{\partial p_{m j}}{\partial r_{m j}}\right|_{r_{m j}=R_{j}}
$$

It can be seen that exponential formula presented by Pedrosa (1986) is applied in this mathematical model. In this paper, the mathematical model is solved based on the analytical method, and the exponential model is selected from the point of view of realization. In fact, other mathematical expressions such as power law model (Bernabe, 1986) and polynomial model (Zhang et al., 2000; Fan et al., 2002) for describing stress sensitivity effect of permeability were introduced.

The permeability modulus $\gamma$ is defined as:

$$
\gamma=\frac{1}{k_{f}} \frac{d k_{f}}{d p_{f}}
$$

From Eq. (12), we can get:

$$
k_{f}=k_{f i} e^{\gamma\left(p_{f}-p_{i}\right)}
$$


In order to eliminate the influence of viscosity $(\mu)$ and compressibility factor $(Z)$, the pseudo pressure is introduced:

$$
\begin{aligned}
& \psi_{m}=\int_{0}^{p_{m}} \frac{2 p}{\mu Z} d p \\
& \psi_{f}=\int_{0}^{p_{f}} \frac{2 p}{\mu Z} d p
\end{aligned}
$$

and permeability modulus can be transformed as follows:

$$
\gamma=\frac{1}{k_{f}} \frac{d k_{f}}{d p_{f}}=\frac{1}{k_{f}} \frac{d k_{f}}{d \psi_{f}} \frac{d \psi_{f}}{d p_{f}}=\frac{1}{k_{f}} \frac{d k_{f}}{d \psi_{f}} \frac{2 p_{f}}{\mu Z}
$$

So the pseudo permeability modulus can be defined as:

$$
\gamma_{p}=\frac{\gamma}{\frac{2 p_{f}}{\mu Z}}=\frac{1}{k} \frac{d k}{d \psi}=\frac{\mu Z}{2 p_{f}} \gamma
$$

Substituting Eqs. (11)-(16) into Eq. (1)-(5) yields:

$$
\begin{aligned}
& \frac{1}{r_{m 1}^{2}} \frac{\partial}{\partial r_{m 1}}\left(r_{m 1}^{2} k_{m 1} \frac{\partial \psi_{m 1}}{\partial r_{m 1}}\right)=\phi_{m 1} \mu_{1} C_{t m 1} \frac{\partial \psi_{m 1}}{\partial t} \\
& \psi_{m 1}\left(r_{m 1}, 0\right)=\psi_{i} \\
& \left.\frac{\partial \psi_{m 1}}{\partial r_{m 1}}\right|_{r_{m 1}=0}=0 \\
& \left.\psi_{m 1}\right|_{r_{m 1}=R_{1}}=\psi_{f 1} \\
& e^{\gamma_{p}\left(\psi_{f 1}-\psi_{i}\right)}\left[\frac{1}{r} \frac{\partial}{\partial r}\left(k_{f h i 1} r \frac{\partial \psi_{f 1}}{\partial r}\right)+\frac{\partial}{\partial z}\left(k_{f v i l} \frac{\partial \psi_{f 1}}{\partial z}\right)\right] \\
& -\left.\frac{3}{R_{1}} k_{m 1} \frac{\partial \psi_{m 1}}{\partial r_{m 1}}\right|_{r_{m 1}=R_{1}}=\phi_{f 1} \mu_{1} C_{t f 1} \frac{\partial \psi_{f 1}}{\partial t}
\end{aligned}
$$

Then the dimensionless variables are presented in Appendix A. According the dimensionless variables, the dimensionless equations can be obtained. To eliminate the nonlinearity of Eq. (22), the perturbation theory is applied. Taking the Laplace transformation with respect to $t_{D}$, taking the finite Fourier cosine transformation with respect to $z_{D}$, the following model of inner region is obtained (see Appendix B):

$$
\frac{\partial^{2} \tilde{\bar{\xi}}_{f 1 D 0}}{\partial r_{D}^{2}}+\frac{1}{r_{D}} \frac{\partial \tilde{\bar{\xi}}_{f 1 D 0}}{\partial r_{D}}=g_{1}^{n} \tilde{\bar{\xi}}_{f 1 D 0}
$$

where:

$$
\begin{gathered}
g_{1}^{n}=f_{1}+n^{2} \pi^{2} L_{1 D}^{2}(n=0,1,2, \ldots) \\
f_{1}=s \omega_{1}+3 \lambda_{1}\left(\sqrt{\frac{\left(1-\omega_{1}\right) s}{\lambda_{1}}} \operatorname{coth} \sqrt{\left.\frac{\left(1-\omega_{1}\right) s}{\lambda_{1}}-1\right)}\right.
\end{gathered}
$$

Similarly, the dimensionless mathematical model of outer region can be obtained as follows:

$$
\frac{1}{r_{m 2}^{2}} \frac{\partial}{\partial r_{m 2}}\left(r_{m 2}^{2} k_{m 2} \frac{\partial \psi_{m 2}}{\partial r_{m 2}}\right)=\phi_{m 2} \mu_{2} C_{t m 2} \frac{\partial \psi_{m 2}}{\partial t}
$$

$$
\begin{gathered}
\psi_{m 2}\left(r_{m 2}, 0\right)=\psi_{i} \\
\left.\frac{\partial \psi_{m 2}}{\partial r_{m 2}}\right|_{r_{m 2}=0}=0
\end{gathered}
$$

$$
\left.\psi_{m 2}\right|_{r_{m 2}=R_{2}}=\psi_{f 2}
$$

$$
\begin{aligned}
& e^{\gamma_{p}\left(\psi_{f 2}-\psi_{i}\right)}\left[\frac{1}{r} \frac{\partial}{\partial r}\left(k_{f h i 2} r \frac{\partial \psi_{f 2}}{\partial r}\right)+\frac{\partial}{\partial z}\left(k_{f v i 2} \frac{\partial \psi_{f 2}}{\partial z}\right)\right] \\
& -\left.\frac{3}{R_{2}} k_{m 2} \frac{\partial \psi_{m 2}}{\partial r_{m 2}}\right|_{r_{m 2}=R_{2}}=\phi_{f 2} \mu_{2} C_{t f 2} \frac{\partial \psi_{f 2}}{\partial t}
\end{aligned}
$$

Substituting the dimensionless variables and perturbation technique into Eqs. (25)-(29), taking the Laplace transformation and the finite Fourier cosine transformation with respect to $t_{D}$ and $z_{D}$ respectively, the following model of outer region is obtained (see Appendix B):

$$
\frac{\partial^{2} \tilde{\bar{\xi}}_{f 2 D 0}}{\partial r_{D}^{2}}+\frac{1}{r_{D}} \frac{\partial \tilde{\bar{\xi}}_{f 2 D 0}}{\partial r_{D}}=g_{2}^{n} \bar{\xi}_{f 2 D 0}
$$

where:

$$
g_{2}^{n}=f_{2}+n^{2} \pi^{2} L_{2 D}^{2}(n=0,1,2, \ldots)
$$

$f_{2}=s \omega_{2} \eta_{12}+3 \lambda_{2}\left(\sqrt{\frac{\left(1-\omega_{2}\right) s \eta_{12}}{\lambda_{2}}} \operatorname{coth} \sqrt{\frac{\left(1-\omega_{2}\right) s \eta_{12}}{\lambda_{2}}}-1\right)$

To solve the above equations, the inner boundary condition, outer boundary condition and two interface conditions are necessary, as Eqs. (33)-(36) showing as follows:

Inner boundary condition:

$$
\lim _{r_{D} \rightarrow 0^{+}} r_{D} \frac{\partial \tilde{\bar{\xi}}_{f 1 D 0}}{\partial r_{D}}=-\frac{\cos \left(n \pi z_{w D}\right)}{2 s L}
$$

Outer boundary condition:

$$
\lim _{r_{D} \rightarrow \infty} \tilde{\bar{\xi}}_{f 2 D 0}=0
$$

Interface conditions:

$$
\tilde{\bar{\xi}}_{f_{1 D 0}}\left(r_{1 D}\right)=\tilde{\tilde{\xi}}_{f 2 D 0}\left(r_{1 D}\right)
$$

$$
\left.\frac{\partial \tilde{\bar{\xi}}_{f 1 D 0}}{\partial r_{D}}\right|_{r_{D}=r_{1 D}}=\left.\frac{1}{M_{12}} \frac{\partial \tilde{\bar{\xi}}_{f 2 D 0}}{\partial r_{D}}\right|_{r_{D}=r_{1 D}}
$$




\section{Model solution}

The formation pressure of the composite TGR can be solved through the combination of Eqs. (22), (30) and (33)(36). The general solution of modified Bessels Eqs. (22) and (30) can be expressed as follows:

$$
\begin{aligned}
& \tilde{\xi}_{f 1 D 0}=A_{1} I_{0}\left(\sqrt{g_{1}^{n}} r_{D}\right)+B_{1} K_{0}\left(\sqrt{g_{1}^{n}} r_{D}\right) \\
& \tilde{\xi}_{f 2 D 0}=A_{2} I_{0}\left(\sqrt{g_{2}^{n}} r_{D}\right)+B_{2} K_{0}\left(\sqrt{g_{2}^{n}} r_{D}\right)
\end{aligned}
$$

Substituting Eq. (38) into the outer boundary condition of Eq. (34), we can get:

$$
\lim _{r_{D} \rightarrow \infty}\left[A_{2} I_{0}\left(\sqrt{g_{2}^{n}} r_{D}\right)+B_{2} K_{0}\left(\sqrt{g_{2}^{n}} r_{D}\right)\right]=0
$$

The coefficient $A_{2}$ can be easily obtained according to the property of Bessel's function as follows:

$$
A_{2}=0
$$

Substituting Eq. (37) into the inner boundary condition of Eq. (34) yields:

$$
\begin{aligned}
& \lim _{r_{D} \rightarrow 0^{+}} r_{D}\left[\sqrt{g_{1}^{n}} A_{1} I_{1}\left(\sqrt{g_{1}^{n}} r_{D}\right)-\sqrt{g_{1}^{n}} B_{1} K_{1}\left(\sqrt{g_{1}^{n}} r_{D}\right)\right] \\
& =-\frac{\hat{q} \cos \left(n \pi z_{w D}\right)}{s q_{s c}}
\end{aligned}
$$

The coefficient $B_{1}$ can be obtained according to the property of Bessel's function as follows:

$$
B_{1}=\frac{\hat{q} \cos \left(n \pi z_{w D}\right)}{s q_{s c}}
$$

According to interface conditions, we get:

$$
\begin{gathered}
A_{1} I_{0}\left(\sqrt{g_{1}^{n}} r_{1 D}\right)+B_{1} K_{0}\left(\sqrt{g_{1}^{n}} r_{1 D}\right) \\
=A_{2} I_{0}\left(\sqrt{g_{2}^{n}} r_{1 D}\right)+B_{2} K_{0}\left(\sqrt{g_{2}^{n}} r_{1 D}\right) \\
A_{1} I_{1}\left(\sqrt{g_{1}^{n}} r_{1 D}\right)-B_{1} K_{1}\left(\sqrt{g_{1}^{n}} r_{1 D}\right) \\
=\frac{\sqrt{g_{2}^{n}}}{M_{12} \sqrt{g_{1}^{n}}}\left[A_{2} I_{1}\left(\sqrt{g_{2}^{n}} r_{1 D}\right)-B_{2} K_{1}\left(\sqrt{g_{2}^{n}} r_{1 D}\right)\right]
\end{gathered}
$$

With the combination of Eqs. (40), (43) and (44), the coefficient $A_{1}$ can be obtained as follows (see Eq. (45)):

$$
A_{1}=\frac{\left(\begin{array}{l}
M_{12} \sqrt{g_{1}^{n}} K_{1}\left(\sqrt{g_{1}^{n}} r_{1 D}\right) K_{0}\left(\sqrt{g_{2}^{n}} r_{1 D}\right) \\
-\sigma_{2}^{n} K_{0}\left(\sqrt{g_{1}^{n}} r_{1 D}\right) K_{1}\left(\sqrt{g_{2}^{n}} r_{1 D}\right)
\end{array}\right)}{\left(\begin{array}{l}
M_{12} \sqrt{g_{1}^{n}} I_{1}\left(\sqrt{g_{1}^{n}} r_{1 D}\right) K_{0}\left(\sqrt{g_{2}^{n}} r_{1 D}\right) \\
+\sigma_{2}^{n} I_{0}\left(\sqrt{g_{1}^{n}} r_{1 D}\right) K_{1}\left(\sqrt{g_{2}^{n}} r_{1 D}\right)
\end{array}\right)} B_{1}
$$

Taking Eqs. (42) and (45) into Eq. (37) yields:

$$
\begin{aligned}
\tilde{\bar{\xi}}_{w D 0} & =\tilde{\xi}_{f 1 D 0} \\
& =\frac{\hat{q} \cos \left(n \pi z_{w D}\right)}{s q_{s c}}\left[\beta_{n} I_{0}\left(\sqrt{g_{1}^{n}} r_{D}\right)+K_{0}\left(\sqrt{g_{1}^{n}} r_{D}\right)\right]
\end{aligned}
$$

where:

$$
\beta_{n}=\frac{\left(\begin{array}{l}
M_{12} \sqrt{g_{1}^{n}} K_{1}\left(\sqrt{g_{1}^{n}} r_{1 D}\right) K_{0}\left(\sqrt{g_{2}^{n}} r_{1 D}\right) \\
-\sqrt{g_{2}^{n}} K_{0}\left(\sqrt{g_{1}^{n}} r_{1 D}\right) K_{1}\left(\sqrt{g_{2}^{n}} r_{1 D}\right)
\end{array}\right)}{\left(\begin{array}{c}
M_{12} \sqrt{g_{1}^{n}} I_{1}\left(\sqrt{g_{1}^{n}} r_{1 D}\right) K_{0}\left(\sqrt{g_{2}^{n}} r_{1 D}\right) \\
+\sqrt{g_{2}^{n}} I_{0}\left(\sqrt{g_{1}^{n}} r_{1 D}\right) K_{1}\left(\sqrt{g_{2}^{n}} r_{1 D}\right)
\end{array}\right)}
$$

Applying the inverse cosine transformation:

$$
\bar{\xi}_{w D 0}=\mathrm{C}_{0}+2 \sum_{n=1}^{\infty} C_{n} \cos \left(n \pi z_{D}\right)
$$

So far the point source solution in inner region is obtained as follows:

$$
\begin{aligned}
& \bar{\xi}_{w D 0}=\frac{1}{2 L s}\left[\beta_{0} I_{0}\left(\sqrt{g_{1}^{0}} r_{D}\right)+K_{0}\left(\sqrt{g_{1}^{0}} r_{D}\right)\right] \\
& +\frac{1}{L s} \sum_{n=1}^{\infty} \cos \left(n \pi z_{w D}\right) \cos \left(n \pi z_{D}\right)\left[\beta_{n} I_{0}\left(\sqrt{g_{1}^{n}} r_{D}\right)+K_{0}\left(\sqrt{g_{1}^{n}} r_{D}\right)\right]
\end{aligned}
$$

By integrating the point source from $-L$ to $L$ (after dimensionless treatment: -1 to 1 ), we can get the pressure distribution in the reservoir for horizontal well which can be taken as the line source in the center of composite TGR. When the horizontal well is completely in the inner region, the wellbore pressure distribution in Laplace domain can be obtained as follows:

$$
\begin{aligned}
& \bar{\xi}_{w D}=\int_{-1}^{1} \frac{1}{2 s}\left[\beta_{0} I_{0}\left(\sqrt{g_{1}^{0}} r_{D}\right)+K_{0}\left(\sqrt{g_{1}^{0}} r_{D}\right)\right] d x_{w D} \\
& +\sum_{n=1}^{\infty} \frac{\cos \left(n \pi z_{w D}\right) \cos \left(n \pi z_{D}\right)}{s} \int_{-1}^{1}\left[\begin{array}{l}
\beta_{n} I_{0}\left(\sqrt{g_{1}^{n}} r_{D}\right) \\
+K_{0}\left(\sqrt{g_{1}^{n}} r_{D}\right)
\end{array}\right] d x_{w D}
\end{aligned}
$$

where $r_{D}=\sqrt{\left(x_{D}-x_{w D}\right)^{2}+\left(y_{D}-y_{w D}\right)^{2}}$.

With the method of Duhamel's principle (Van Everdingen and Hurst, 1949), the solution considering well storage effect and skin effect can be obtained as follows:

$$
\bar{\xi}_{w D}\left(s, C_{D}, S\right)=\frac{s \bar{\xi}_{w D}(s)+\frac{S}{2 L_{1 D}}}{s+C_{D} s^{2}\left[s \bar{\xi}_{w D}(s)+\frac{S}{2 L_{1 D}}\right]}
$$

By means of Stehfest numerical inversion algorithm (Stehfest, 1970), the perturbation solution $\xi_{w D}\left(r_{D}, t_{D}\right)$ in real space can be solved, thus the transient pressure of wellbore in real space is expressed as follows:

$$
\psi_{w D}=-\frac{1}{\gamma_{p D}} \ln \left[1-\gamma_{p D} \xi_{w D}\left(r_{D}, t_{D}\right)\right]
$$

From the research achievement of Van Everdingenet and Hurst (1949) when the well is producing at a constant bottomhole pressure, the dimensionless rate in Laplace domain can be calculated as follows:

$$
\bar{Q}_{D}=\frac{1}{s^{2} \bar{\psi}_{w D}}
$$




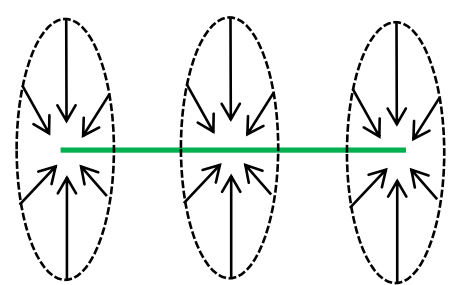

(a) First radial flow

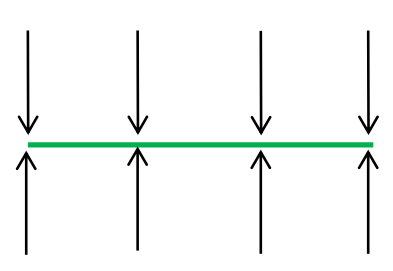

(b) Linear flow

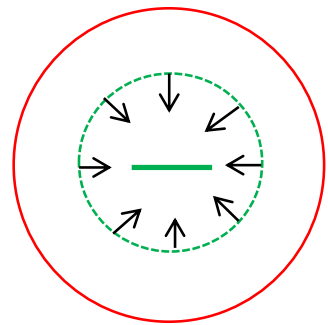

(c) Second radial flow

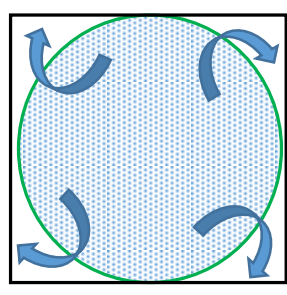

(d) Transfer regime

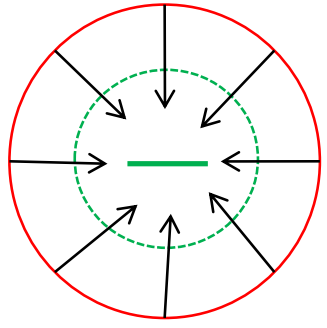

(e) Late pseudo-radial flow

Fig. 3. Schematic of flow regimes.

So after obtaining the dimensionless pseudo pressure solution, taking the numerical Laplace transform (Yao et al., 2008) over the numerical inversion results $\psi_{w D}$, then the dimensionless rate can be solved with Eq. (54) and Stehfest numerical inversion algorithm. The numerical Laplace transform can be expressed as follows:

$$
\begin{gathered}
\bar{\psi}_{w D}(s)=\frac{1}{s^{2}}\left\{\begin{array}{l}
\psi_{w D}^{\prime}(1)\left[1-e^{-s t_{D}(1)}\right] \\
+\sum_{i=2}^{n-1} \psi_{w D}^{\prime}(i)\left[e^{-s t_{D}(i-1)}-e^{-s t_{D}(i)}\right] \\
+\psi_{w D}^{\prime}(n) e^{-s t_{D}(n)}
\end{array}\right\} \\
\psi_{w D}^{\prime}(i)=\frac{\psi_{w D}(i)-\psi_{w D}(i-1)}{t_{D}(i)-t_{D}(i-1)}
\end{gathered}
$$

\section{Results and discussion}

\subsection{Type curves and interpretation}

The code is programed by Matlab2017b. The numerical integration, Stehfest numerical inversion algorithm are used for wellbore pressure and rate solutions in real space. The type curves of composite TGR with stress-sensitivity effect are shown in Figs. 3-5. Fig. 3 shows some classical flow regimes. Fig. 4 and Fig. 5 show all flow regimes.

Different flow regimes are identified based on the type curves.

Regime 1: The early wellbore storage period. The slope is 1 in pressure and pressure derivative curves in log-log coordinates, and in rate and rate derivative curves the slope becomes -1 .

Regime 2: The skin factor period. This period is influenced by the value of skin factor. The pressure derivative curves are shown as convex.

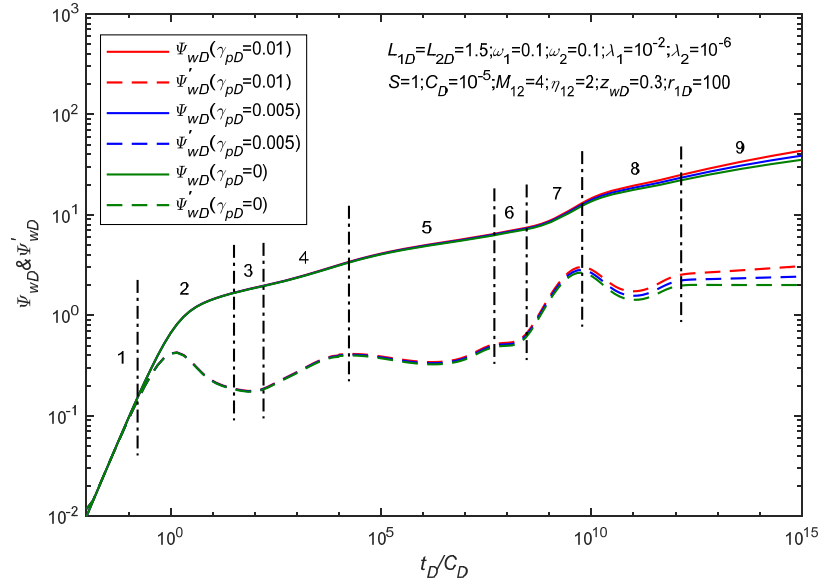

Fig. 4. Pressure-transient-type curves of horizontal well.

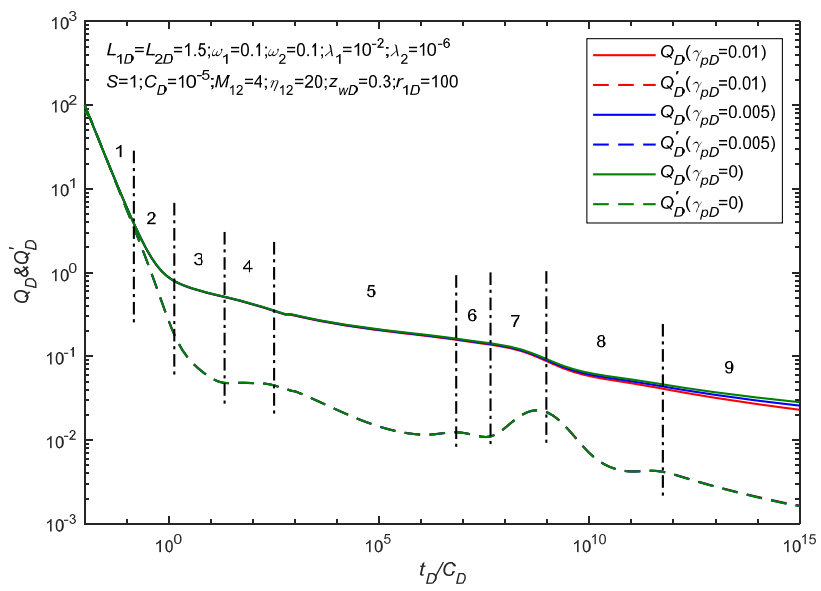

Fig. 5. Rate-transient-type curves of horizontal well. 
Regime 3: The first radial flow regime. In this regime, the pressure derivative curve is shown as a level straight line, the value of the level straight line is about $1 /\left(4 L_{1 D}\right)$. The production rate derivation curve is shown as a drawdown straight line. The gas in this period flows to wellbore radially in the vertical plane. It will last until the pressure wave spreads to top/bottom boundary.

Regime 4: The linear flow regime. The pressure derivative curve manifests as a straight line with a slope of 0.5. The production rate derivative curve is shown as a level straight line. This period reflects the linear flow feature around horizontal wellbore.

Regime 5: The transfer regime in inner region. In this regime both of the pressure derivative and production rate derivative curves are manifested as the concave shape. This period reflects that the cross flow between the matrix and fractures systems in inner region begins.

Regime 6: The second radial flow regime. The pressure derivative curve is shown as a level straight line, the value of the level straight line is 0.5 . The production rate derivation curve is shown as a drawdown straight line.

Regime 7: Transition regime. It means the fluid of outer region starts to flow to the inner region.

Regime 8: The transfer regime in outer region. In this regime both of the pressure derivative and production rate derivative curves manifest as the concave shape, which is a typical characteristic for dual porosity media. The gas will flow from matrix to fractures.

Regime 9: Late pseudo-radial flow regime. Following regime 8 , this regime starts when the gas flow from matrix to fractures and that from fractures to wellbore reach a dynamic balance state. Due to the stress sensitivity, the pressure derivative curves appear to be upward. If the stress sensitivity is ignored, the value in pressure derivative curves is constant which is $M_{12} / 2$, and the production rate and rate derivative curves are parallel.

\subsection{Effect of relevant parameters}

In this section, the influences of some relevant parameters are discussed. During the discussion, one of the parameters is changed while other parameters are controlled, and a series of curves can be obtained to analyze.

(1) Effect of permeability modulus: Fig. 4 shows the effect of permeability modulus $\gamma_{p D}$ on PTA-type curves. As can be seen in Fig. 4, when $\gamma_{p D}$ increases, i.e., the stronger the stresssensitivity effect is, the greater the degree of upward. With the increase of permeability modulus, the permeability of fracture system becomes lower. Thus it's more difficult to flow in the formation for natural gas, which leads to lager drawdown pressure in TGR. Fig. 5 shows the effect of permeability modulus on RTA-type curves, from which we can see the stronger stress-sensitivity effect causes lower production rate for TGR.

(2) Effect of skin factor: Fig. 6 manifests the effect of skin factor $S$ on PTA-type curves. The skin factor mainly affects regime 2 . When the value of $S$ is 0 , the convex shape disappe-

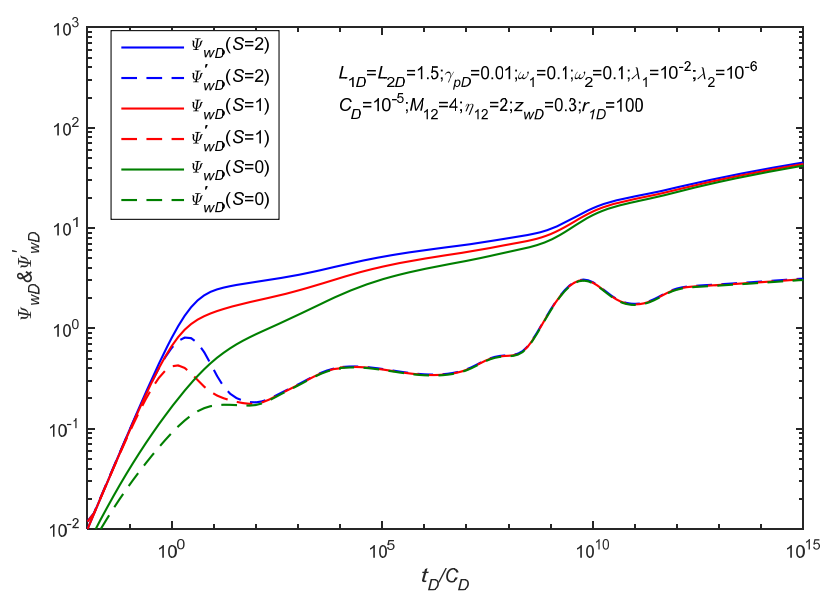

Fig. 6. The effect of skin factor on pressure-transient-type curves.

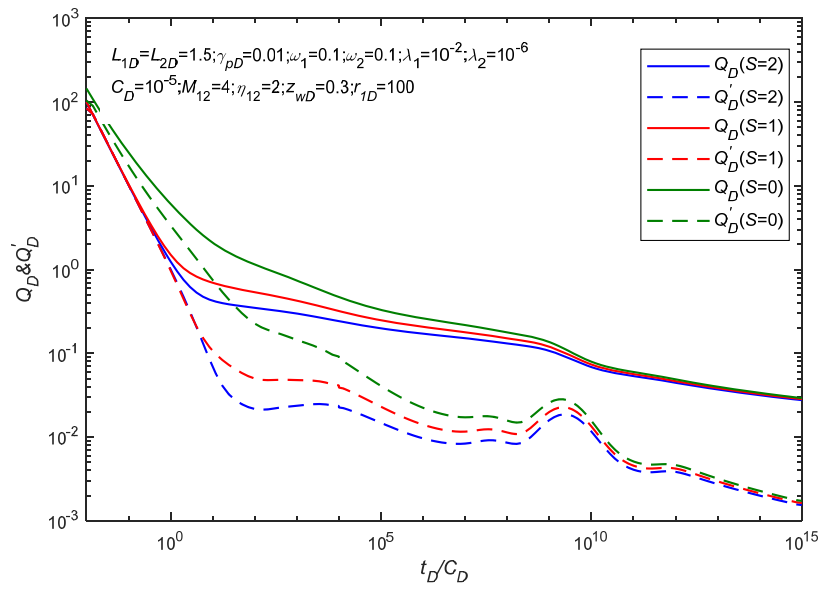

Fig. 7. The effect of skin factor on rate-transient-type curves.

ars. When the skin factor increases, the resistance around wellbore will increase, engendering greater difficulty for gas stream to flow into the wellbore. Fig. 7 reflects the effect of skin factor on RTA-type curves. The additional resistance caused by skin factor will lead to the decrease of production rate.

(3) Effect of inner region storage coefficient: Fig. 8 shows the effect of inner region storage coefficient $\omega_{1}$ on PTA-type curves. The inner region storage coefficient mainly affects regime 3 , regime 4 and regime 5 . When the value of $\omega_{1}$ increases, the duration of regime 3 increases at the same time, the starting time of regime 4 and regime 5 will be later. And the bigger the $\omega_{1}$ is, the shorter time transfer regime lasts, the narrower and shallower the concave shape is. The similar results can be seen in RTA-type curves (Fig. 9). The inner region storage coefficient $\omega_{1}$ reflects the storage capacity of fracture system. The bigger value of $\omega_{1}$ indicates bigger storage capacity of fractures and smaller storage capacity of matrix respectively. When $\omega_{1}$ increases, the original stored fluid in the fracture system flow to wellbore will last longer, and the quantity of exchange between matrix and fracture systems will be smaller. 


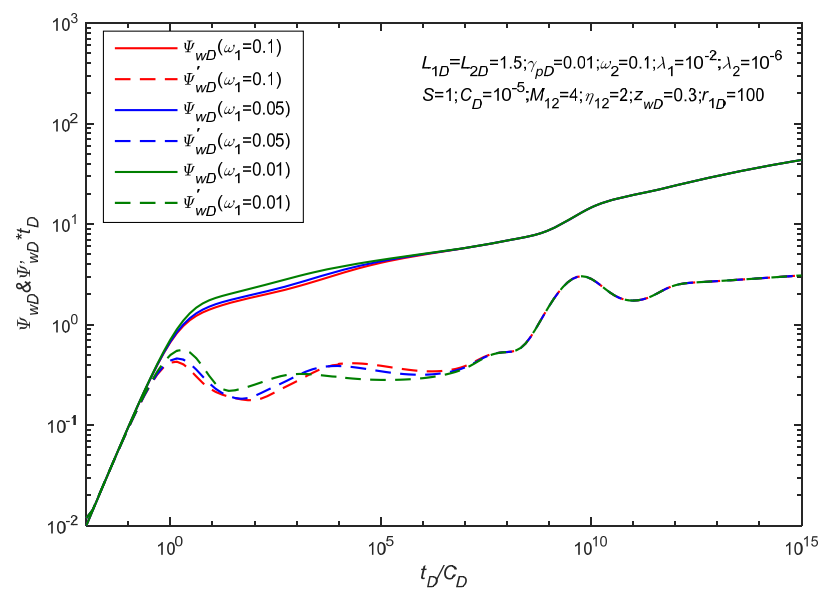

Fig. 8. The effect of inner region transfer coefficient on pressure-transienttype curves.

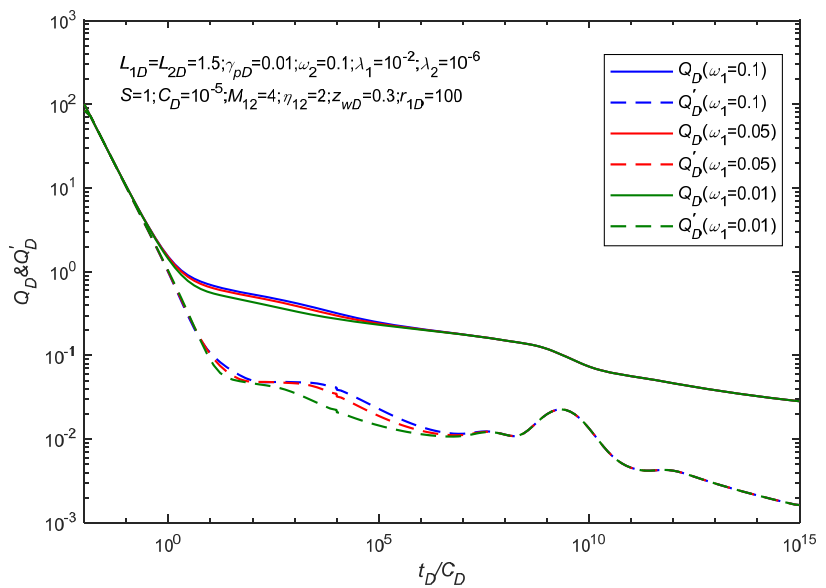

Fig. 9. The effect of inner region transfer coefficient on rate-transient-type curve.

(4) Effect of outer region storage coefficient: The influence of outer region storage coefficient $\omega_{2}$ on pressure and prodction rate is shown in Figs. 10 and 11, respectively. It's apprarent that the outer region storage coefficient mainly affects regime 7 and regime 8 . With the increase of the parameter $\omega_{2}$, the transient regime lasts longer, therefore the transfer regime of outer regime emerges later, the concave shape is narrower and shallower at the same time. This result is similar to part (3). So we can conclude that outer region storage coefficient dominate the intensity of cross flow.

(5) Effect of inner region transfer coefficient: Fig. 12 indicates the effect of inner region transfer coefficient $\lambda_{1}$ on PTAtype curves. It has been shown that the inner region transfer coefficient $\lambda_{1}$ mainly affects regime 5 and regime 6 . A bigger transfer coefficient $\lambda_{1}$ results in relatively earlier beginning time of regime 5 and longer time of regime 6 . The second radial flow period may be absent when $\lambda_{1}$ is too small. The smaller transfer coefficient leads to the transfer regime occours more difficultly. From Fig. 13 we can notice that bigger inner region transfer coefficient leads to bigger production rate from production rate curves, and earlier beginning time of the concave shape form production rate derivative curves. The parameter $\lambda_{1}$ means the difficulty level of the gas releasing from matrix. When $\lambda_{1}$ increases, more natural gas

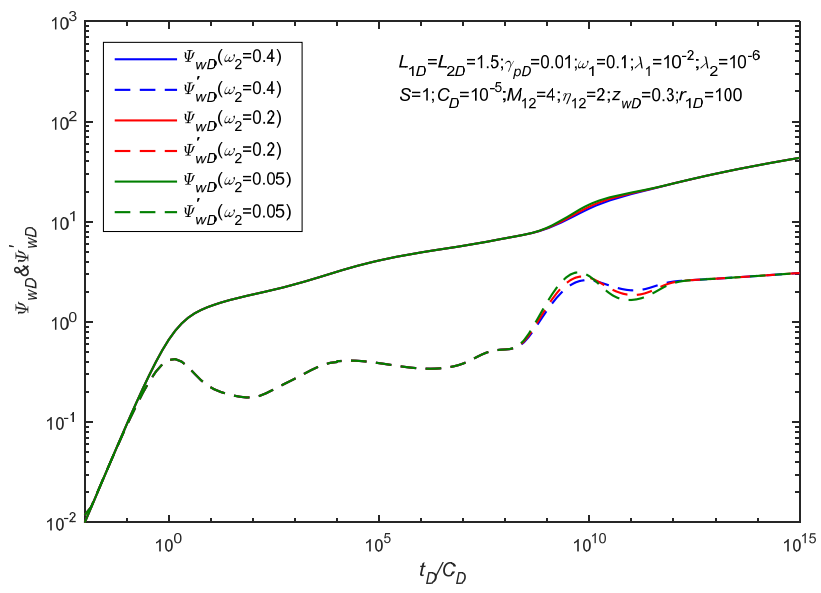

Fig. 10. The effect of outer region storage coefficient on pressure-transienttype curves.

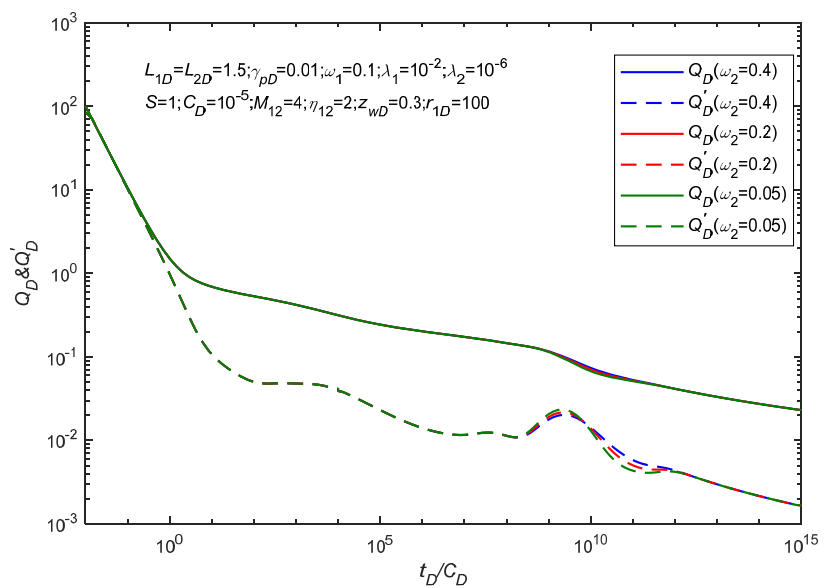

Fig. 11. The effect of outer region storage coefficient on rate-transient-type curves.

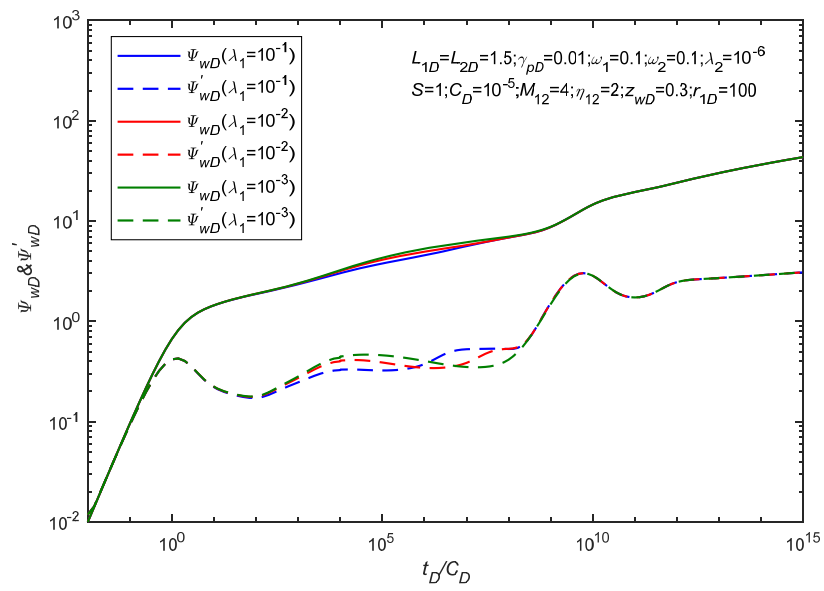

Fig. 12. The effect of inner region transfer coefficient on pressure-transienttype curves. 


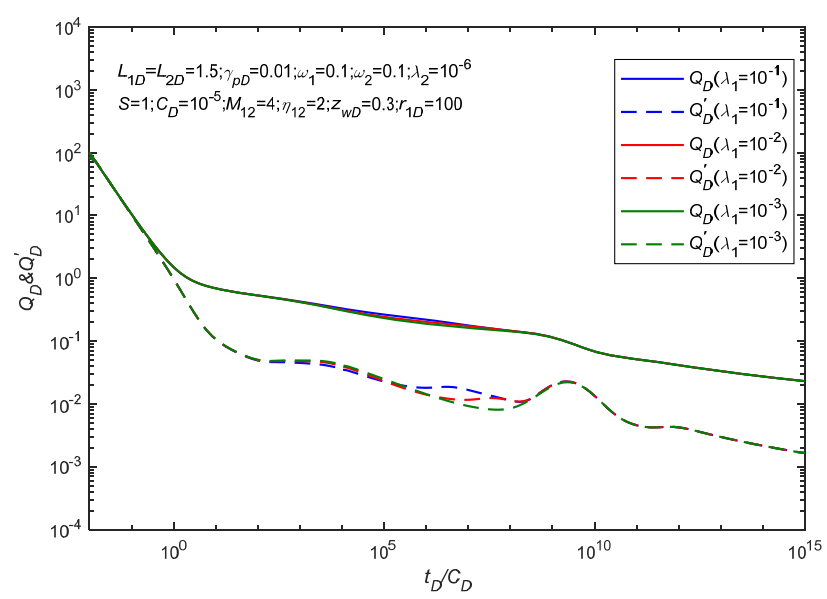

Fig. 13. The effect of inner region transfer coefficient on rate-transient-type curves.

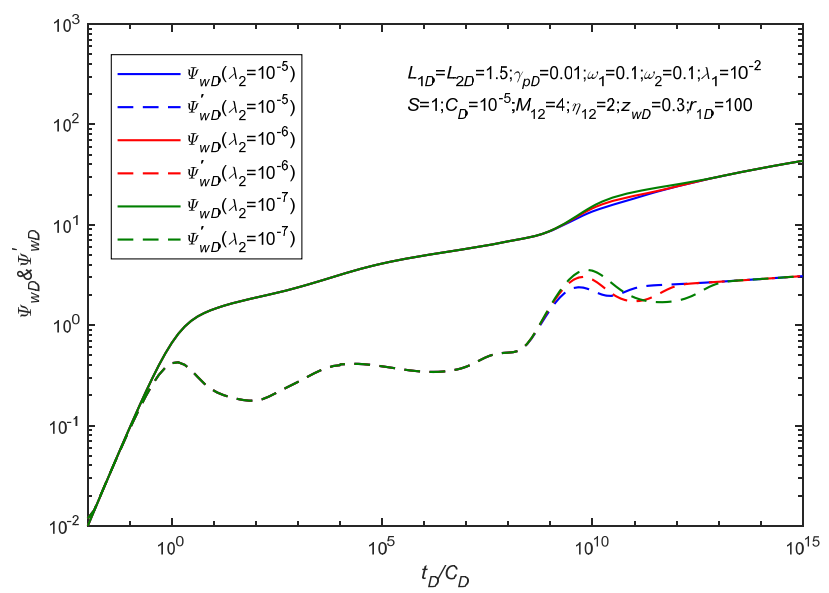

Fig. 14. The effect of outer region transfer coefficient on pressure-transienttype curves.

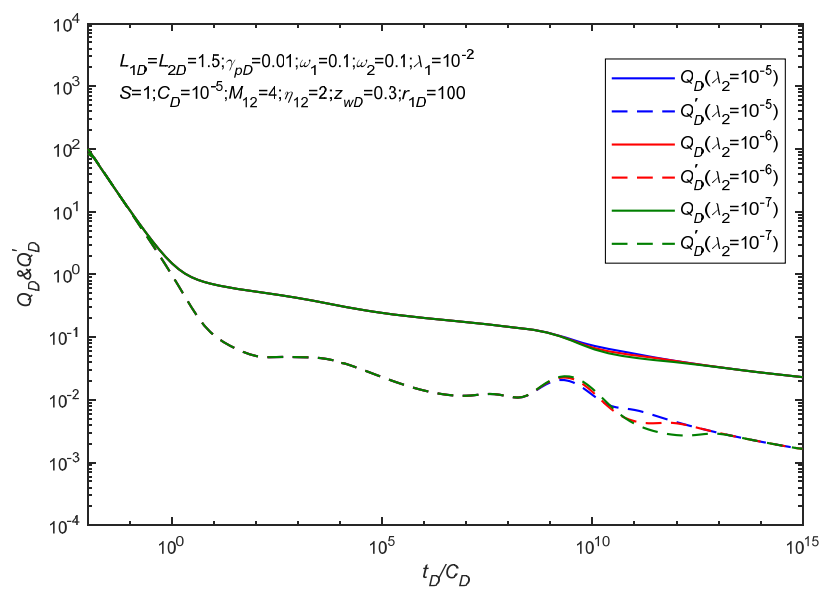

Fig. 15. The effect of outer region transfer coefficient on rate-transient-type curves.

can be produced from formation, so the production rate in corresponding regime ascends.

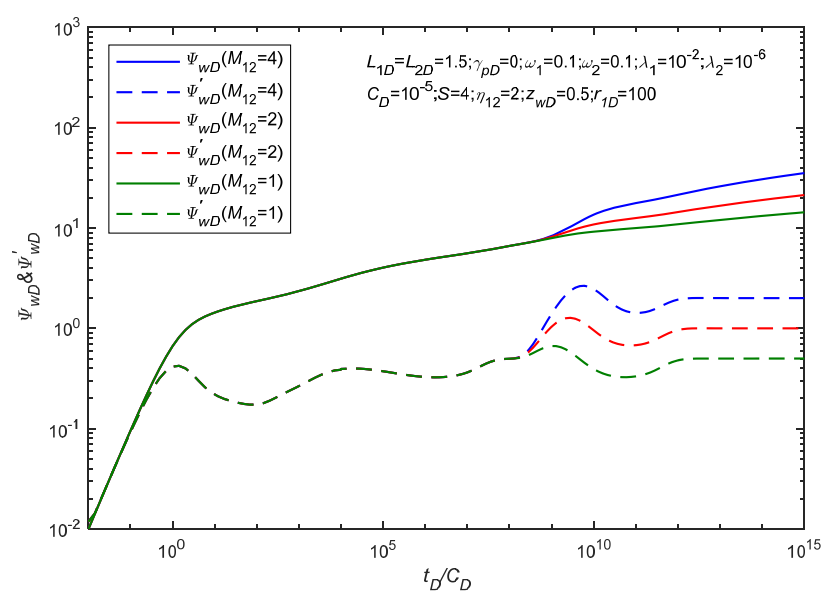

Fig. 16. The effect of mobility ratio on pressure-transient-type curves.

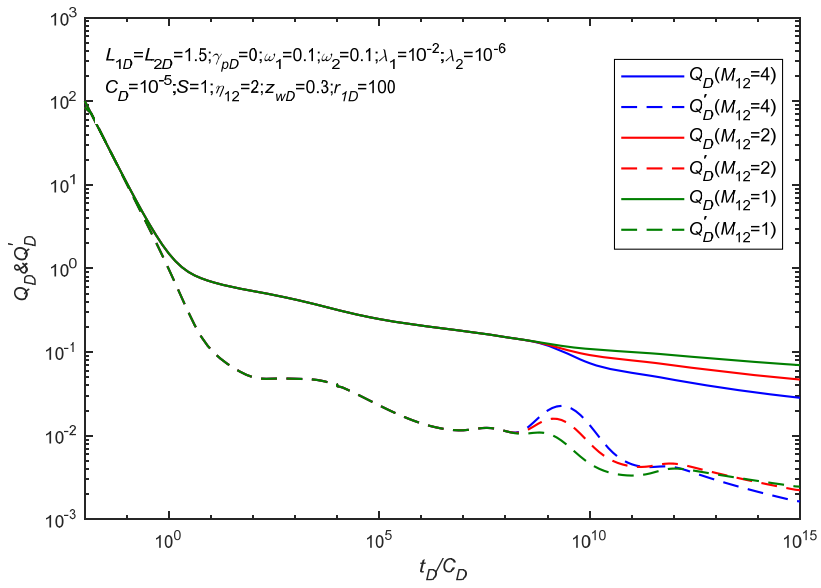

Fig. 17. The effect of mobility ratio on rate-transient-type curves.

(6) Effect of outer region transfer coefficient: Figs. 14 and 15 reflects the effect of outer region transfer coefficient $\lambda_{2}$ on PTA-type curves and RTA-type curves respectively. From the figures we can see the parameter $\lambda_{2}$ mainly affects regime 7 and regime 8 . The results in outer region is akin to the results obtained in inner region. Hence the parameter $\lambda_{2}$ dominates the duration of regime 8 and regime 9 , it also affects the production rate.

(7) Effect of mobility ratio: Fig. 16 shows the effect of the mobility ratio $M_{12}$ on PTA-type curves. The mobility ratio mainly affects regime 7 , regime 8 and regime 9 . When the mobility ratio increases, the transient regime lasts longer therefore regime 8 and regime 9 emerges later. Bigger mobility ratio means it is more difficult for fluid to flow in outer region, which results in the extension of transient period. On the other hand, in fact, the value of the horizontal line of pressure derivative curves in regime 9 is about $M_{12} / 2$, that's why the pressure derivative curves go upward when mobility ratio increases. From Fig. 17 we can notice that bigger mobility ratio leads to smaller production rate in regime 7 to regime 9 .

(8) Effect of inner region radius: Fig. 18 shows the effect 


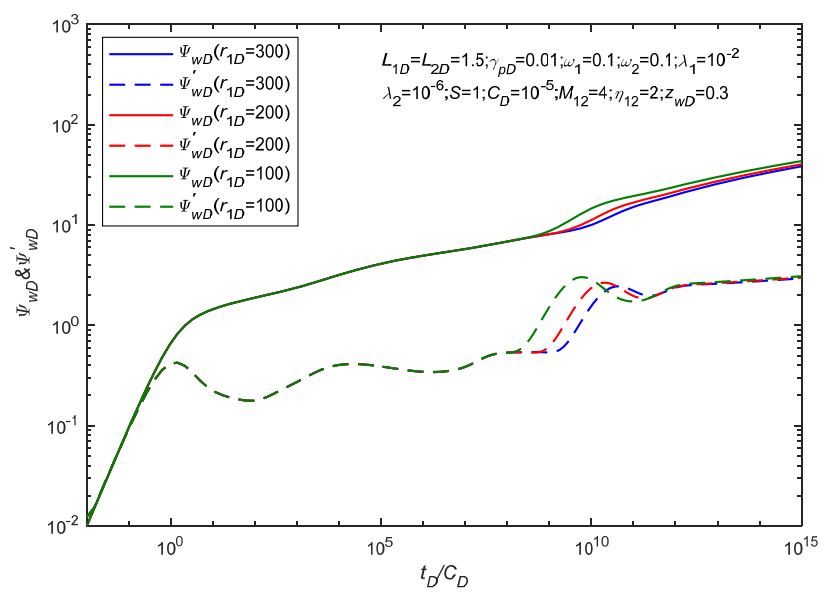

Fig. 18. The effect of inner region radius on pressure-transient-type curves.

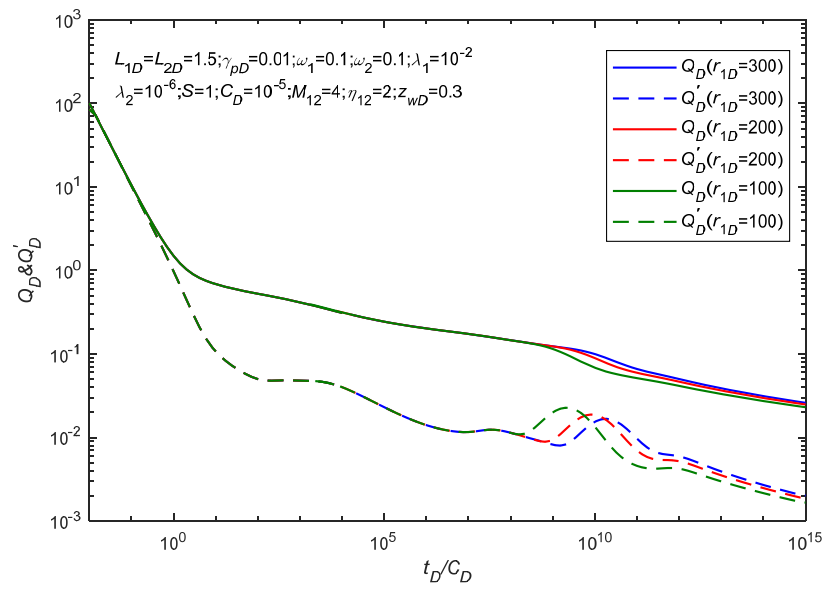

Fig. 19. The effect of inner region radius on rate-transient-type curves.

of inner region radius $r_{1 D}$ on PTA-type curves. It can be seen that inner region radius mainly affects the regimes after second radial flow regime. It's apparent that if $r_{1 D}$ increases, the longer time to take for the pressure wave to reach the outer region, so the regime 7 and regime 8 occurs later. The smaller the radius of the inner region, the smaller the pressure loss at the boundary of the inner and outer regions. Hence from Fig. 19 we can notice the production rate will increases if inner region radius decreases.

(9) Effect of horizontal well position in vertical direction: Fig. 20 manifests the effect of the parameter $z_{w D}$ on PTAtype curves. Seen from Fig. 20, the horizontal well position in vertical direction mainly affects the first radial flow regime. When the horizontal well position in vertical direction $z_{w D}$ decreases, i.e. the distance between the horizontal well and the upper/bottom boundary decreases, the first radial flow regime lasts shorter. Seen from Fig. 21, when the horizontal well position in vertical direction $z_{w D}$ decreases, the production rate will decrease in all time for well production lifetime, but the difference is not apparent.

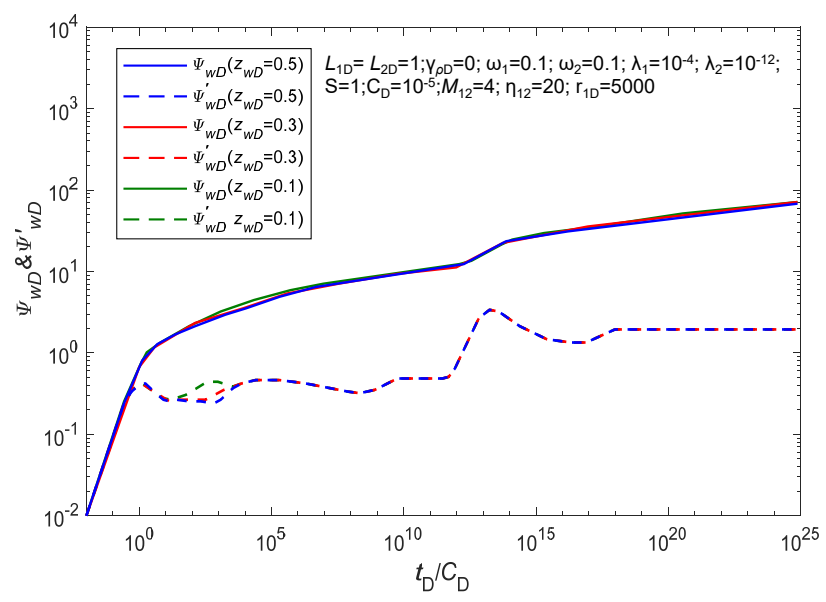

Fig. 20. The effect of horizontal well position in vertical direction on pressuretransient-type curves.

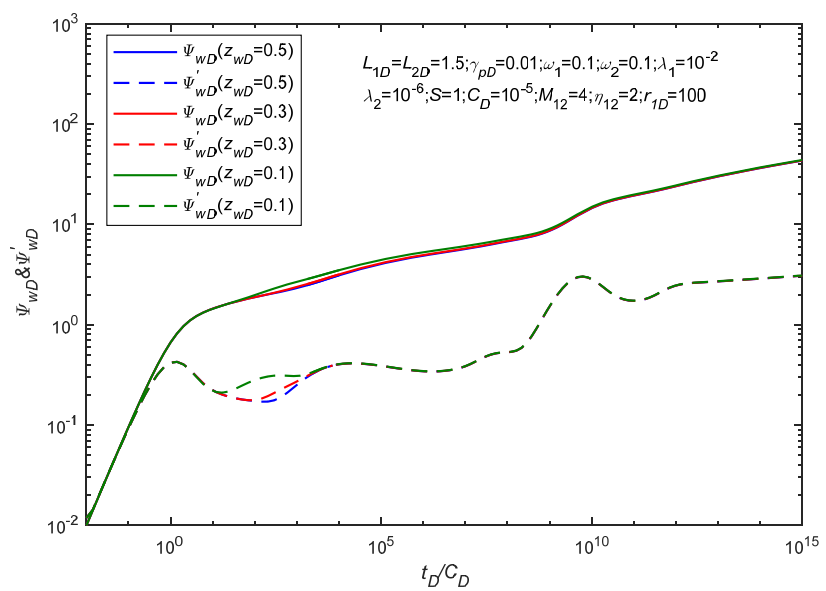

Fig. 21. The effect of horizontal well position in vertical direction on ratetransient-type curves.

\section{Conclusions}

This paper deduced a mathematical model which considers the transport mechanisms for tight gas including the unsteady state cross flow, stress-sensitive effect, well storage effect and skin effect. The results showed different flow regimes and the influence of related factors on pressure and production performance. From the above analysis, the following conclusions can be summarized:

1) Type curves can be obtained through Stehfest numerical inversion algorithm. Nine flow regimes are identified based on the type curves including early wellbore storage period, skin effect regime, first radial flow regime, linear flow regime, transfer regime in inner region, second radial flow regime, transition regime, transfer regime in outer region, and late-pseudo radial flow regime.

2) In transfer regimes both of the pressure derivative and production rate derivative curves manifest as the concave shape. The stress-sensitivity effect leads to the upward of pressure derivative curves and downward of rate curves. When ignoring stress-sensitivity effect, the value in pres- 
sure derivative curves is constant which is $M_{12} / 2$, and the production rate and rate derivative curves are parallel.

3) The existence of stress-sensitivity effect leads to the increase of pressure drawdown and the decrease of production rate. Storage coefficient mainly influences the intensity of cross flow. Transfer coefficient has significant effects on the duration of transfer regimes. Horizontal well position in vertical direction and inner region radius have significant effects on first radial flow regime and second radial flow regime respectively.

\section{Nomenclature}

\section{Latin symbols}

$C=$ Wellbore storage coefficient, $\mathrm{m}^{3} / \mathrm{Pa}$

$C_{t}=$ Total compressibility coefficient, $\mathrm{Pa}^{-1}$

$h=$ Reservoir thickness, $\mathrm{m}$

$k=$ Permeability, $\mathrm{m}^{2}$

$L=$ Half-length of horizontal well, $\mathrm{m}$

$M=$ Mobility radio, dimensionless

$n=$ Natural number $(\mathrm{n}=0,1,2, \cdots)$

$p=$ Pressure, $\mathrm{Pa}$

$Q=$ Production rate, $\mathrm{m}^{3} / \mathrm{s}$

$R=$ Radius of matrix block, $\mathrm{m}$

$r=$ Radial distance, $\mathrm{m}$

$S=$ Skin factor, dimensionless

$s=$ Laplace transform variable

$T=$ Absolute temperature, $\mathrm{K}$

$t=$ Time, second

$x=$ Horizontal distance, $\mathrm{m}$

$y=$ Horizontal distance, $\mathrm{m}$

$Z=$ Gas deviation factor, dimensionless

$z=$ Vertical distance, $\mathrm{m}$

\section{Greek symbols}

$\varepsilon=$ Tiny variable

$\gamma=$ Permeability modulus, $\mathrm{Pa}^{-1}$

$\omega=$ Storage coefficient, dimensionless

$\lambda=$ Transfer coefficient, dimensionless

$\eta=$ Diffusion coefficient ratio, dimensionless

$\mu=$ Viscosity, $\mathrm{Pa} \cdot \mathrm{s}$

$\phi=$ Porosity, fraction

$\psi=$ Pseudo pressure, $\mathrm{Pa}^{2} /(\mathrm{Pa} \cdot \mathrm{s})$

$\zeta=$ Perturbation deformation function

$\xi_{0}=$ Zero-order perturbation deformation function

\section{Superscripts}

- = Laplace transform domain

$\sim$ Finite Fourier transform domain

' = Derivative

\section{Subscripts}

$1=$ Inner region

$2=$ Outer region

$$
\begin{aligned}
& D=\text { Dimensionless } \\
& f=\text { Fracture system } \\
& h=\text { Horizontal } \\
& i=\text { Initial } \\
& { }_{m}=\text { Matrix system } \\
& s c=\text { Standard condition } \\
& { }_{t}=\text { Total } \\
& { }_{v}=\text { Vertical } \\
& { }_{w}=\text { Wellbore }
\end{aligned}
$$

Open Access This article is distributed under the terms and conditions of the Creative Commons Attribution (CC BY-NC-ND) license, which permits unrestricted use, distribution, and reproduction in any medium, provided the original work is properly cited.

\section{References}

Albinali, A., Holy, R., Sarak, H., et al. Modeling of 1D anomalous diffusion in fractured nanoporous media. Oil Gas Sci. Technol. 2016, 71(4): 56.

Brown, M., Ozkan, E., Raghavan, R., et al. Practical solutions for pressure-transient responses of fractured horizontal wells in unconventional shale reservoirs. SPE Reserv. Eval. Eng. 2011, 14(6): 663-676.

Chang, J., Yortsos, Y.C. Pressure transient analysis of fractal reservoirs. SPE Form. Eval. 1990, 5(1): 31-38.

de Swaan, A. Pressure transients in a fractal-cluster model of porous media. Oil Gas Sci. Technol. 2016, 71(1): 9.

de Swaan, O.A. Analytic solutions for determining naturally fractured reservoir properties by well testing. Soc. Petrol. Eng. J. 1976, 16(3): 117-122.

Ding, D.Y., Farah, N., Bourbiaux, B., et al. Simulation of matrix/fracture interaction in low-permeability fractured unconventional reservoirs. SPE J. 2018, 23(4): 13891411.

Ezulike, O., Igbokoyi, A. Horizontal well pressure transient analysis in anisotropic composite reservoirs-A three dimensional semi-analytical approach. J. Pet. Sci. Eng. 2012, 96: 120-139.

Freeman, C.M., Moridis, G.J., Blasingame, T.A. A numerical study of microscale flow behavior in tight gas and shale gas reservoir systems. Transp. Porous Media 2011, 90(1): 253-268.

Göktas, B., Ertekin, T. Performances of openhole completed and cased horizontal/undulating wells in thin-bedded, tight sand gas reservoirs. Paper SPE 65619 Presented at SPE Eastern Regional Meeting, Morgantown, West Virginia, 17-19 October, 2000.

Holditch, S.A. Tight gas sands. J. Pet. Technol. 2006, 58(6): 86-93.

Jia, C., Zheng, M., Zhang, Y. Unconventional hydrocarbon resources in China and the prospect of exploration and development. Pet. Explor. Dev. 2012, 39(2): 139-146.

Jiang, R., Gao, Y., Sun, Z., et al. Rate transient analysis for horizontal well passing through inner region of composite gas reservoir. Natural Gas Geoscience 2015, 34(2): 8185. (in Chinese)

Kazemi, H. Pressure transient analysis of naturally fractured reservoirs with uniform fracture distribution. Soc. Petrol. 
Eng. J. 1969, 9(4): 451-462.

Kuchuk, F.J., Biryukov, D., Fitzpatrick, T. Rate transient and decline curve analyses for continuously (dual-porosity) and discretely naturally fractured reservoir. Paper SPE 170698 Presented at SPE Annual Technical Conference and Exhibition, Amsterdam, The Netherlands, 27-29 October, 2014.

Li, X., Cao, L., Luo, C., et al. Characteristics of transient production rate performance of horizontal well in fractured tight gas reservoirs with stress-sensitivity effect. J. Pet. Sci. Eng. 2017, 158: 92-106.

Naik, G.C. Tight gas reservoirsan unconventional natural energy source for the future. Accessed on 3 April 2007.

Ngo, T.D., Fourno, A., Noetinger, B. Modeling of transport processes through large-scale discrete fracture networks using conforming meshes and open-source software. J. Hydrol. 2017, 554: 66-79.

Nie, R., Meng, Y., Jia, Y., et al. Dual porosity and dual permeability modeling of horizontal well in naturally fractured reservoir. Transp. Porous Media 2012, 92(1): 213-235.

Noetinger, B. A quasi steady state method for solving transient Darcy flow in complex 3D fractured networks accounting for matrix to fracture flow. J. Comput. Phys. 2015, 283: 205-223.

Ozkan, E., Raghavan, R., Joshi, S.D. Horizontal well pressure analysis. Paper SPE 16378 Presented at SPE California Regional Meeting, Ventura, California, 8-10 April, 1987.

Pedrosa Jr, O.A. Pressure transient response in stresssensitive formations. Paper SPE 151155 Presented at SPE California Regional Meeting, Oakland, California, 2-4 April, 1986.

Shi, G., Nie, R., Lu, J., et al. Well test model of horizontal well in 2-zoned composite reservoir and example interpretation. Journal of Southwest Petroleum University (Science \& Technology Edition) 2012, 34(5): 99-106. (in Chinese)
Shi, Y., Sun, X. Stress sensitivity analysis of Changqing tight clastic reservoir. Pet. Explor. Dev. 2001, 28(5): 85-87.

Stehfest, H. Algorithm 368: Numerical inversion of Laplace transforms [D5]. Commun. ACM 1970, 13(1): 47-49.

Van Everdingen, A.F., Hurst, W. The application of the Laplace transformation to flow problems in reservoirs. J. Pet. Technol. 1949, 1(12): 305-324.

Wang, X., Liu, C. Pressure analysis for horizontal wells in composite. Acta Petrolei Sinica 1997, 18: 72-77. (in Chinese)

Warren, J.E., Root, P.J. The behavior of naturally fractured reservoirs. Soc. Petrol. Eng. J. 1963, 3(3): 245-255.

Wu, Y., Li, J., Ding, D., et al. A generalized framework model for the simulation of gas production in unconventional gas reservoirs. SPE J. 2014, 19(5): 845-857.

Xu, B., Haghighi, M., Li, X., et al. Development of new type curves for production analysis in naturally fractured shale gas/tight gas reservoirs. J. Pet. Sci. Eng. 2013, 105: 107-115.

Xu, J., Guo, C., Wei, M., et al. Production performance analysis for composite shale gas reservoir considering multiple transport mechanisms. J. Nat. Gas Sci. Eng. 2015, 26: 382-395.

Yu, Z., Xiong, W., Gao, S., et al. Stress sensitivity of tight reservoir and its influence on oilfield development. Acta Petrolei Sinica 2007, 4: 95-98. (in Chinese)

Zhang, H., Kang, Y., Chen, Y., et al. Deformation theory and stress sensitivity of tight sandstones reserviors. Natural Gas Geoscience 2004, 15(5): 482-485. (in Chinese)

Zhang, W., Jiang, R., Xu, J., et al. Production performance analysis for horizontal wells in composite coal bed methane reservoir. Energy Explor. Exploit. 2017, 35(2): 194-217.

Zhang, W., Xu, J., Jiang, R., et al. Employing a quad-porosity numerical model to analyze the productivity of shale gas reservoir. J. Pet. Sci. Eng. 2017, 157: 1046-1055. 


\section{Appendix A. Dimensionless definition}

Table A-1. Dimensionless definition formulas.

\begin{tabular}{llc}
\hline Dimensionless variables & Inner region & Outer region \\
\hline Dimensionless matrix pseudo pressure & $\psi_{m 1 D}=\frac{k_{f h i 1} h\left(\psi_{i}-\psi_{m 1}\right)}{Q_{s c} \mu_{1}}$ & $\psi_{m 2 D}=\frac{k_{f h i 1} h\left(\psi_{i}-\psi_{m 2}\right)}{Q_{s c} \mu_{1}}$ \\
Dimensionless fracture pseudo pressure & $\psi_{f 1 D}=\frac{k_{f h i 1} h\left(\psi_{i}-\psi_{f 1}\right)}{Q_{s c} \mu_{1}}$ & $\psi_{f 2 D}=\frac{k_{f h i} h\left(\psi_{i}-\psi_{f 2}\right)}{Q_{s c} \mu_{1}}$ \\
Transfer coefficient & $\lambda_{1}=\frac{k_{m 1} L^{2}}{k_{f h i 1} R_{1}^{2}}$ & $\lambda_{2}=\frac{k_{m 2} L^{2}}{k_{f h i 2} R_{2}^{2}}$ \\
Storage coefficient & $\omega_{1}=\frac{\phi_{f 1} C_{t f 1}}{\left(\phi_{1} C_{t 1}\right)_{f+m}}$ & $\omega_{2}=\frac{\phi_{f 2} C_{t f 2}}{\left(\phi_{2} C_{t 2}\right)_{f+m}}$ \\
Dimensionless wellbore length & $L_{1 D}=\frac{L}{h} \sqrt{\frac{k_{f v i 1}}{k_{f h i 1}}}$ & $L_{2 D}=\frac{L}{h} \sqrt{\frac{k_{f v i 2}}{k_{f h i 2}}}$ \\
Dimensionless time & & $t_{D}=\frac{k_{f h i 1}}{\left(\phi_{1} C_{t 1}\right)_{f+m} \mu_{1} L^{2}} t$ \\
Dimensionless wellbore storage coefficient & & $C_{D}=\frac{C}{2 \pi\left(\phi_{1} C_{t 1}\right)_{f+m} h L^{2}}$ \\
Mobility ratio & $M_{12}=\frac{k_{f h i 1} / \mu_{1}}{k_{f h i 2} / \mu_{2}}$ \\
Diffusion coefficient ratio & $\eta_{12}=\frac{k_{f h i 1} /\left(\phi_{1} \mu_{1} C_{t 1}\right)_{f+m}}{k_{f h i 2} /\left(\phi_{2} \mu_{2} C_{t 2}\right)_{f+m}}$ \\
Dimensionless pseudo permeability modulus & $\gamma_{p D}=\frac{q_{s c} \mu_{1}}{k_{f h i 1} h} \gamma_{p}$ \\
Dimensionless length & $r_{m D}=\frac{r_{m}}{R}, r_{D}=\frac{r}{L}, x_{D}=\frac{x}{L}, y_{D}=\frac{y}{L}, z_{D}=\frac{z}{h}, \varepsilon_{D}=\frac{\varepsilon}{h}$ \\
\hline
\end{tabular}

\section{Appendix B. Derivation of mathematical model}

\section{Inner region}

Mathematical model of matrix system in inner region can be shown as follows:

$$
\left\{\begin{array}{l}
\operatorname{lr} \frac{1}{r_{m 1}^{2}} \frac{\partial}{\partial r_{m 1}}\left(r_{m 1}^{2} k_{m 1} \frac{\partial \psi_{m 1}}{\partial r_{m 1}}\right)=\phi_{m 1} \mu_{1} C_{m 1} \frac{\partial \psi_{m 1}}{\partial t} \\
\psi_{m 1}\left(r_{m 1}, 0\right)=\psi_{i} \\
\left.\frac{\partial \psi_{m 1}}{\partial r_{m 1}}\right|_{r_{m 1}=0}=0 \\
\left.\psi_{m 1}\right|_{r_{m 1}=R_{1}}=\psi_{f 1}
\end{array}\right.
$$

Substituting dimensionless variables into Eq. (B-1), the dimensionless flow model of matrix system can be expressed as:

$$
\left\{\begin{array}{l}
\operatorname{lr} \frac{\partial^{2} \psi_{m 1 D}}{\partial r_{m 1 D}}+\frac{2}{r_{m 1 D}} \frac{\partial \psi_{m 1 D}}{\partial r_{m 1 D}}=\frac{1-\omega_{1}}{\lambda_{1}} \frac{\partial \psi_{m 1 D}}{\partial t_{D}} \\
\psi_{m 1 D}\left(r_{m 1 D}, 0\right)=0 \\
\left.\frac{\partial \psi_{m 1 D}}{\partial r_{m 1 D}}\right|_{r_{m 1 D}=0}=0 \\
\left.\psi_{m 1 D}\right|_{r_{m 1 D}=R_{1 D}}=\psi_{f 1 D}
\end{array}\right.
$$

Taking following Laplace transform:

$$
\bar{P}\left(r_{D}, z_{D}, s\right)=\int_{0}^{\infty} P\left(r_{D}, z_{D}, t_{D}\right) e^{-s t_{D}} d t_{D}
$$


The dimensionless mathematical model in Laplace space can be simplified as follows:

$$
\left\{\begin{array}{l}
\operatorname{lr} \frac{\partial^{2} \bar{\psi}_{m 1 D}}{\partial r_{m 1 D}{ }^{2}}+\frac{2}{r_{m 1 D}} \frac{\partial \bar{\psi}_{m 1 D}}{\partial r_{m 1 D}}=\frac{\left(1-\omega_{1}\right) s}{\lambda_{1}} \bar{\psi}_{m 1 D} \\
\bar{\psi}_{m 1 D}\left(r_{m 1 D}, 0\right)=0 \\
\left.\frac{\partial \bar{\psi}_{m 1 D}}{\partial r_{m 1 D}}\right|_{r_{m 1 D}=0}=0 \\
\left.\bar{\psi}_{m 1 D}\right|_{r_{m 1 D}=R_{1 D}}=\bar{\psi}_{f 1 D}
\end{array}\right.
$$

The solution of Eq. (B-4) is obtained:

$$
\bar{\psi}_{m 1 D}=\left[\frac{\sinh \left(\sqrt{\frac{\left(1-\omega_{1}\right) s}{\lambda_{1}}} r_{m 1 D}\right)}{r_{m 1 D} \sinh \left(\sqrt{\frac{\left(1-\omega_{1}\right) s}{\lambda_{1}}}\right)}\right] \bar{\psi}_{f 1 D}
$$

For fracture system in inner region, the differential equation expressed as follows:

$$
e^{\gamma_{p}\left(\psi_{f 1}-\psi_{i}\right)}\left[\frac{1}{r} \frac{\partial}{\partial r}\left(k_{f h i 1} r \frac{\partial \psi_{f 1}}{\partial r}\right)+\frac{\partial}{\partial z}\left(k_{f v i 1} \frac{\partial \psi_{f 1}}{\partial z}\right)\right]-\left.\frac{3}{R_{1}} k_{m 1} \frac{\partial \psi_{m 1}}{\partial r_{m 1}}\right|_{r_{m 1}=R_{1}}=\phi_{f 1} \mu_{1} C_{t f 1} \frac{\partial \psi_{f 1}}{\partial t}
$$

Substituting dimensionless variables into Eq. (B-6) yields:

$$
e^{-\gamma_{p D} \psi_{f 1 D}}\left(\frac{\partial^{2} \psi_{f 1 D}}{\partial r_{D}^{2}}+\frac{1}{r_{D}} \frac{\partial \psi_{f 1 D}}{\partial r_{D}}+L_{1 D}^{2} \frac{\partial^{2} \psi_{f 1 D}}{\partial z_{D}^{2}}\right)-\left.3 \lambda_{1} \frac{\partial \psi_{m 1 D}}{\partial r_{m 1 D}}\right|_{r_{m 1 D}=1}=\omega_{1} \frac{\partial \psi_{f 1 D}}{\partial t_{D}}
$$

The Pedrosa (1986) presented a variable substitution technique to eliminate the nonlinearity of PDE, the substitution formula is:

$$
\psi_{f D}=-\frac{1}{\gamma_{p D}} \ln \left[1-\gamma_{p D} \xi_{f D}\left(r_{D}, t_{D}\right)\right]
$$

Taking the perturbation theory, which can be expressed as follows:

$$
\begin{gathered}
\xi_{f D}=\xi_{f D 0}+\gamma_{p D} \xi_{f D 1}+\gamma_{p D}^{2} \xi_{f D 2}+\cdots \\
\frac{1}{1-\gamma_{p D} \xi_{f D}}=1+\gamma_{D} \xi_{\beta D}+\left(\gamma_{p D} \xi_{f D}\right)^{2}+\left(\gamma_{p D} \xi_{f D}\right)^{3}+\cdots \\
-\frac{1}{\gamma_{p D}} \ln \left[1-\gamma_{p D} \xi_{f D}\left(r_{D}, t_{D}\right)\right]=-\frac{1}{\gamma_{p D}}\left[-\gamma_{p D} \xi_{f D}-\frac{1}{2}\left(\gamma_{p D} \xi_{f D}\right)^{2}-\frac{1}{3}\left(\gamma_{p D} \xi_{f D}\right)^{3}-\cdots\right]
\end{gathered}
$$

Substituting Eqs. (B-8)-(B-11) into Eq. (B-7), and employing zero-order approximate solution (the dimensionless permeability modulus is far less than 1), yields:

$$
\frac{\partial^{2} \xi_{f 1 D 0}}{\partial r_{D}^{2}}+\frac{1}{r_{D}} \frac{\partial \xi_{f 1 D 0}}{\partial r_{D}}+L_{1 D}^{2} \frac{\partial^{2} \xi_{f 1 D 0}}{\partial z_{D}^{2}}-\left.3 \lambda_{1} \frac{\partial p_{m 1 D}}{\partial r_{m 1 D}}\right|_{r_{m 1 D}=1}=\omega_{1} \frac{\partial \xi_{f 1 D 0}}{\partial t_{D}}
$$

Taking the Laplace transform over dimensionless time $t_{D}$ and substituting Eq. (B-5) into Eq. (B-12), we obtain:

$$
\frac{\partial^{2} \bar{\xi}_{f 1 D 0}}{\partial r_{D}{ }^{2}}+\frac{1}{r_{D}} \frac{\partial \bar{\xi}_{f 1 D 0}}{\partial r_{D}}+L_{1 D}^{2} \frac{\partial^{2} \bar{\xi}_{f 1 D 0}}{\partial z_{D}^{2}}=f_{1} \bar{\xi}_{f 1 D 0}
$$

where:

$$
f_{1}=s \omega_{1}+3 \lambda_{1}\left[\sqrt{\frac{\left(1-\omega_{1}\right) s}{\lambda_{1}}} \operatorname{coth}\left(\sqrt{\frac{\left(1-\omega_{1}\right) s}{\lambda_{1}}}\right)-1\right]
$$

Taking the finite Fourier cosine transform over $z_{D}$, yields:

$$
\tilde{\bar{P}}=\int_{0}^{1} \bar{P}\left(r_{D}, z_{D}, s\right) \cos \left(n \pi z_{D}\right) d z_{D} \quad(n=0,1,2, \ldots)
$$


yields:

$$
\frac{\partial^{2} \tilde{\bar{\xi}}_{f 1 D 0}}{\partial r_{D}^{2}}+\frac{1}{r_{D}} \frac{\partial \tilde{\xi}_{f 1 D 0}}{\partial r_{D}}=g_{1}^{n} \tilde{\bar{\xi}}_{f 1 D 0}
$$

where:

$$
g_{1}^{n}=f_{1}+n^{2} \pi^{2} L_{1 D}^{2} \quad(n=0,1,2, \ldots)
$$

\section{Outer region}

Mathematical model of matrix system in outer region can be shown as follows:

$$
\left\{\begin{array}{l}
\operatorname{lr} \frac{1}{r_{m 2}^{2}} \frac{\partial}{\partial r_{m 2}}\left(r_{m 2}^{2} k_{m 2} \frac{\partial \psi_{m 2}}{\partial r_{m 2}}\right)=\phi_{m 2} \mu_{2} C_{t m 2} \frac{\partial \psi_{m 2}}{\partial t} \\
\psi_{m 2}\left(r_{m 2}, 0\right)=\psi_{i} \\
\left.\frac{\partial \psi_{m 2}}{\partial r_{m 2}}\right|_{r_{m 2}=0}=0 \\
\left.\psi_{m 2}\right|_{r_{m 2}=R_{2}}=\psi_{f 2}
\end{array}\right.
$$

Substituting dimensionless variables into Eq. (B-18), the dimensionless flow model of matrix system can be expressed as follows:

$$
\left\{\begin{array}{l}
\operatorname{lr} \frac{\partial^{2} \psi_{m 2 D}}{\partial r_{m 2 D}{ }^{2}}+\frac{2}{r_{m 2 D}} \frac{\partial \psi_{m 2 D}}{\partial r_{m 2 D}}=\frac{\left(1-\omega_{2}\right) \eta_{12}}{\lambda_{1}} \frac{\partial \psi_{m 2 D}}{\partial t_{D}} \\
\psi_{m 2 D}\left(r_{m 2 D}, 0\right)=0 \\
\left.\frac{\partial \psi_{m 2 D}}{\partial r_{m 2 D}}\right|_{r_{m 2 D}=0}=0 \\
\left.\psi_{m 2 D}\right|_{r_{2 D}=R_{2 D}}=\psi_{f 2 D}
\end{array}\right.
$$

The dimensionless mathematical model in Laplace space can be simplified as follows:

$$
\left\{\begin{array}{l}
\operatorname{lr} \frac{\partial^{2} \bar{\psi}_{m 2 D}}{\partial r_{m 2 D}{ }^{2}}+\frac{2}{r_{m 2 D}} \frac{\partial \bar{\psi}_{m 2 D}}{\partial r_{m 2 D}}=\frac{\left(1-\omega_{2}\right) \eta_{12} s}{\lambda_{2}} \bar{\psi}_{m 2 D} \\
\bar{\psi}_{m 2 D}\left(r_{m 2 D}, 0\right)=0 \\
\left.\frac{\partial \bar{\psi}_{m 2 D}}{\partial r_{m 2 D}}\right|_{r_{m 2 D}=0}=0 \\
\left.\bar{\psi}_{m 2 D}\right|_{r_{2 D}=R_{2 D}}=\bar{\psi}_{f 2 D}
\end{array}\right.
$$

The solution of Eq. (B-20) is obtained as follows:

$$
\bar{\psi}_{m 2 D}=\left[\frac{\sinh \left(\sqrt{\frac{\left(1-\omega_{2}\right) \eta_{12} s}{\lambda_{2}}} r_{m 2 D}\right)}{r_{m 2 D} \sinh \left(\sqrt{\frac{\left(1-\omega_{2}\right) \eta_{12} s}{\lambda_{2}}}\right)}\right] \bar{\psi}_{f 2 D}
$$

For fracture system in outer region, the differential equation expressed as follows:

$$
e^{\gamma_{p}\left(\psi_{f 2}-\psi_{i}\right)}\left[\frac{1}{r} \frac{\partial}{\partial r}\left(k_{f h i 2} r \frac{\partial \psi_{f 2}}{\partial r}\right)+\frac{\partial}{\partial z}\left(k_{f v i 2} \frac{\partial \psi_{f 2}}{\partial z}\right)\right]-\left.\frac{3}{R_{1}} k_{m 2} \frac{\partial \psi_{m 2}}{\partial r_{m 2}}\right|_{r_{m 2}=R_{2}}=\phi_{f 2} \mu_{2} C_{t f 2} \frac{\partial \psi_{f 2}}{\partial t}
$$

Substituting dimensionless variables into Eq. (B-22) yields:

$$
e^{-\gamma_{p D} \psi_{f 2 D}}\left(\frac{\partial^{2} \psi_{f 2 D}}{\partial r_{D}{ }^{2}}+\frac{1}{r_{D}} \frac{\partial \psi_{f 2 D}}{\partial r_{D}}+L_{2 D}^{2} \frac{\partial^{2} \psi_{f 2 D}}{\partial z_{D}^{2}}\right)-\left.3 \lambda_{2} \frac{\partial \psi_{m 2 D}}{\partial r_{m 2 D}}\right|_{r_{m 2 D}=1}=\omega_{2} \eta_{12} \frac{\partial \psi_{f 2 D}}{\partial t_{D}}
$$


Taking the perturbation theory, yields:

$$
\frac{\partial^{2} \xi_{f 2 D 0}}{\partial r_{D}^{2}}+\frac{1}{r_{D}} \frac{\partial \xi_{f 2 D 0}}{\partial r_{D}}+L_{2 D}^{2} \frac{\partial^{2} \xi_{f 2 D 0}}{\partial z_{D}^{2}}-\left.3 \lambda_{2} \frac{\partial p_{m 2 D}}{\partial r_{m 2 D}}\right|_{r_{m 2 D}=1}=\omega_{2} \eta_{12} \frac{\partial \xi_{f 2 D 0}}{\partial t_{D}}
$$

Taking the Laplace transform over dimensionless time $t_{D}$ and substituting Eq. (B-21) into Eq. (B-24), we obtain:

$$
\frac{\partial^{2} \bar{\xi}_{f 2 D 0}}{\partial r_{D}{ }^{2}}+\frac{1}{r_{D}} \frac{\partial \bar{\xi}_{f 2 D 0}}{\partial r_{D}}+L_{2 D}^{2} \frac{\partial^{2} \bar{\xi}_{f 2 D 0}}{\partial z_{D}^{2}}=f_{2} \bar{\xi}_{f 2 D 0}
$$

where:

$$
f_{2}=s \omega_{2} \eta_{12}+3 \lambda_{2}\left[\sqrt{\frac{\left(1-\omega_{2}\right) s \eta_{12}}{\lambda_{2}}} \operatorname{coth}\left(\sqrt{\frac{\left(1-\omega_{2}\right) s \eta_{12}}{\lambda_{2}}}\right)-1\right]
$$

Taking the finite Fourier cosine transform over $z_{D}$, yields:

$$
\frac{\partial^{2} \tilde{\bar{\xi}}_{f 2 D 0}}{\partial r_{D}^{2}}+\frac{1}{r_{D}} \frac{\partial \tilde{\bar{\xi}}_{f 2 D 0}}{\partial r_{D}}=g_{2}^{n} \bar{\xi}_{f 2 D 0}
$$

where:

$$
g_{2}^{n}=f_{2}+n^{2} \pi^{2} L_{2 D}^{2} \quad(n=0,1,2, \ldots)
$$

\section{Additional conditions}

To solve the above equations, the inner boundary condition, outer boundary condition and two interface conditions are necessary.

Inner boundary condition is described as:

$$
\left.\lim _{\varepsilon \rightarrow 0^{+}} \int_{z_{w}-\varepsilon / 2}^{z_{w}+\varepsilon / 2}\left[e^{\gamma_{p}\left(\psi_{f 1}-\psi_{i}\right)} r \frac{\partial \psi_{f 1}}{\partial r}\right] d z_{w}\right|_{r=\varepsilon}=\frac{p_{s c} T Q_{s c}}{2 \pi k_{f h i 1} T_{s c} L},\left|z-z_{w}\right| \leq \varepsilon / 2
$$

Top and bottom boundary conditions:

$$
\begin{aligned}
& \left.\frac{\partial \psi_{f 1}}{\partial z}\right|_{z=h}=\left.\frac{\partial \psi_{f 2}}{\partial z}\right|_{z=h}=0 \\
& \left.\frac{\partial \psi_{f 1}}{\partial z}\right|_{z=0}=\left.\frac{\partial \psi_{f 2}}{\partial z}\right|_{z=0}=0
\end{aligned}
$$

Infinite outer boundary condition:

$$
\lim _{r \rightarrow \infty} \psi_{f 2}(r, t)=\psi_{i}
$$

Interface conditions:

$$
\begin{aligned}
\psi_{f 1}\left(r_{1}, t\right) & =\psi_{f 2}\left(r_{1}, t\right) \\
\left.k_{f h i 1} e^{\gamma_{p}\left(\psi_{f 1}-\psi_{i}\right)} \frac{\partial \psi_{f 1}}{\partial r}\right|_{r=r_{1}} & =\left.k_{f h i 2} e^{\gamma_{p}\left(\psi_{f 2}-\psi_{i}\right)} \frac{\partial \psi_{f 2}}{\partial r}\right|_{r=r_{1}}
\end{aligned}
$$


The dimensionless equations can be obtained as:

$$
\left\{\begin{array}{l}
\left.\lim _{\varepsilon_{D} \rightarrow 0^{+}} \int_{z_{w D}-\varepsilon_{D} / 2}^{z_{w D}+\varepsilon_{D} / 2}\left(e^{-\gamma_{p D} \psi_{f 1 D}} r_{D} \frac{\partial \psi_{f 1 D}}{\partial r_{D}}\right) d z_{w}\right|_{r_{D}=\varepsilon_{D}}=-\frac{1}{2 L},\left|z_{D}-z_{w D}\right| \leq \varepsilon_{D} / 2 \\
\left.\frac{\partial \psi_{f 1 D}}{\partial z_{D}}\right|_{z_{D}=1}=\left.\frac{\partial \psi_{f 2 D}}{\partial z_{D}}\right|_{z_{D}=1}=0 \\
\left.\frac{\partial \psi_{f 1 D}}{\partial z_{D}}\right|_{z_{D}=0}=\left.\frac{\partial \psi_{f 2 D}}{\partial z_{D}}\right|_{z_{D}=0}=0 \\
\lim _{r_{D} \rightarrow \infty} \psi_{f 2 D}\left(r_{D}, t_{D}\right)=0 \\
\psi_{f 1 D}\left(r_{1 D}, t_{D}\right)=\psi_{f 2 D}\left(r_{1 D}, t_{D}\right) \\
\left.e^{-\gamma_{p D} \psi_{f 1 D}} \frac{\partial \psi_{f 1 D}}{\partial r_{D}}\right|_{r_{D}=r_{1 D}}=\left.\frac{1}{M_{12}} e^{-\gamma_{p D} \psi_{f 2 D}} \frac{\partial \psi_{f 2 D}}{\partial r_{D}}\right|_{r_{D}=r_{1 D}}
\end{array}\right.
$$

Taking perturbation technique, Laplace transform and finite Fourier cosine transform, yields:

$$
\left\{\begin{array}{l}
\lim _{r_{D} \rightarrow 0^{+}} r_{D} \frac{\partial \tilde{\bar{\xi}}_{f 1 D 0}}{\partial r_{D}}=-\frac{\cos \left(n \pi z_{w D}\right)}{2 s L} \\
\lim _{r_{D} \rightarrow \infty} \tilde{\bar{\xi}}_{f 2 D 0}=0 \\
\tilde{\bar{\xi}}_{f 1 D 0}\left(r_{1 D}\right)=\tilde{\bar{\xi}}_{f 2 D 0}\left(r_{1 D}\right) \\
\left.\frac{\partial \tilde{\xi}_{f 1 D 0}}{\partial r_{D}}\right|_{r_{D}=r_{i D}}=\left.\frac{1}{M_{12}} \frac{\partial \tilde{\xi}_{f 2 D 0}}{\partial r_{D}}\right|_{r_{D}=r_{i D}}
\end{array}\right.
$$

